\title{
Optimal Quantum Thermometry with Coarse-Grained Measurements
}

\author{
Karen V. Hovhannisyan $\odot,{ }^{1, * *, *}$ Mathias R. Jørgensen $\odot, 2,{ }^{2 *}, \dagger$ Gabriel T. Landi, ${ }^{3,}{ }^{* *},+$ \\ Álvaro M. Alhambra $\odot,{ }^{4, * *, \S}$ Jonatan B. Brask ${ }^{2,},{ }^{2,}$ and Martí Perarnau-Llobet ${ }^{5, \|}$ \\ ${ }^{1}$ The Abdus Salam International Centre for Theoretical Physics (ICTP), Strada Costiera 11, 34151 Trieste, Italy \\ ${ }^{2}$ Department of Physics, Technical University of Denmark, 2800 Kongens Lyngby, Denmark \\ ${ }^{3}$ Instituto de Física da Universidade de São Paulo, São Paulo, 05314-970, Brazil \\ ${ }^{4}$ Max-Planck-Institut für Quantenoptik, 85748 Garching, Germany \\ ${ }^{5}$ Département de Physique Appliquée, Université de Genève, 1211 Geneva, Switzerland
}

(Received 2 December 2020; revised 26 March 2021; accepted 21 April 2021; published 19 May 2021)

\begin{abstract}
Precise thermometry for quantum systems is important to the development of new technology, and understanding the ultimate limits to precision presents a fundamental challenge. It is well known that optimal thermometry requires projective measurements of the total energy of the sample. However, this is infeasible in even moderately sized systems, where realistic energy measurements will necessarily involve some coarse-graining. Here we explore the precision limits for temperature estimation when only coarsegrained measurements are available. Using tools from signal processing, we derive the structure of optimal coarse-grained measurements and find that good temperature estimates can generally be attained even with a small number of outcomes. We apply our results to many-body systems and nonequilibrium thermometry. For the former, we focus on interacting spin lattices, both at and away from criticality, and find that the Fisher-information scaling with system size is unchanged after coarse-graining. For the latter, we consider a probe of given dimension interacting with the sample, followed by a measurement of the probe. We derive an upper bound on arbitrary, nonequilibrium strategies for such probe-based thermometry and illustrate it for thermometry on a Bose-Einstein condensate using an atomic quantum-dot probe.
\end{abstract}

DOI: 10.1103/PRXQuantum.2.020322

\section{INTRODUCTION}

Thermometry is a basic metrological task that is vital throughout science and technology. Estimating temperature is important on all scales, ranging from astronomical bodies with temperatures in the millions of kelvins to atomic systems near absolute zero. In particular, applications of thermometry in nanoscale or microscale devices are becoming increasingly relevant as technology advances [1-4]. Examples include accurate temperature estimation of ultracold gases [5-9], electrons in superconductors [10-12], and the use of atomic-size devices,

\footnotetext{
*khovhann@ictp.it

†matrj@fysik.dtu.dk

$\ddagger$ gtlandi@if.usp.br

§alvaro.alhambra@mpq.mpg.de

ๆjonatan.brask@fysik.dtu.dk

"marti.perarnaullobet@unige.ch

${ }^{* *}$ These authors contributed equally to this work.
}

Published by the American Physical Society under the terms of the Creative Commons Attribution 4.0 International license. Further distribution of this work must maintain attribution to the author(s) and the published article's title, journal citation, and DOI. such as color centers in diamond or quantum dots, as probes to be used in a variety of systems [13-16]. At these scales, quantum effects have a significant influence on the achievable precision. It is therefore important to understand what the fundamental limits for temperature estimation in quantum systems are.

Quantum features offer both advantages and challenges to thermometry $[3,17]$. The advantages range from measurement enhancements due to strong coupling $[8,18,19]$, correlated probes $[20,21]$, or nonequilibrium probes [2229]. The challenges are related to the inherent difficulty of accessing information in quantum systems, due, for instance, to measurement backaction or natural limitations in performing high-resolution measurements [30,31].

When the measurement resolution is unlimited, the ultimate precision of temperature estimation allowed by quantum mechanics is obtained by performing projective measurements of energy [32-34]. However, for large (or even moderately sized) many-body systems, one seldom has access to measurements that distinguish individual energy levels. Instead, one usually measures only a local subsystem of the sample $[19,35,36]$ or performs a global measurement with only finite resolution [30,31] [see Fig. 1(a)]; alternatively, one addresses the sample indirectly, by 

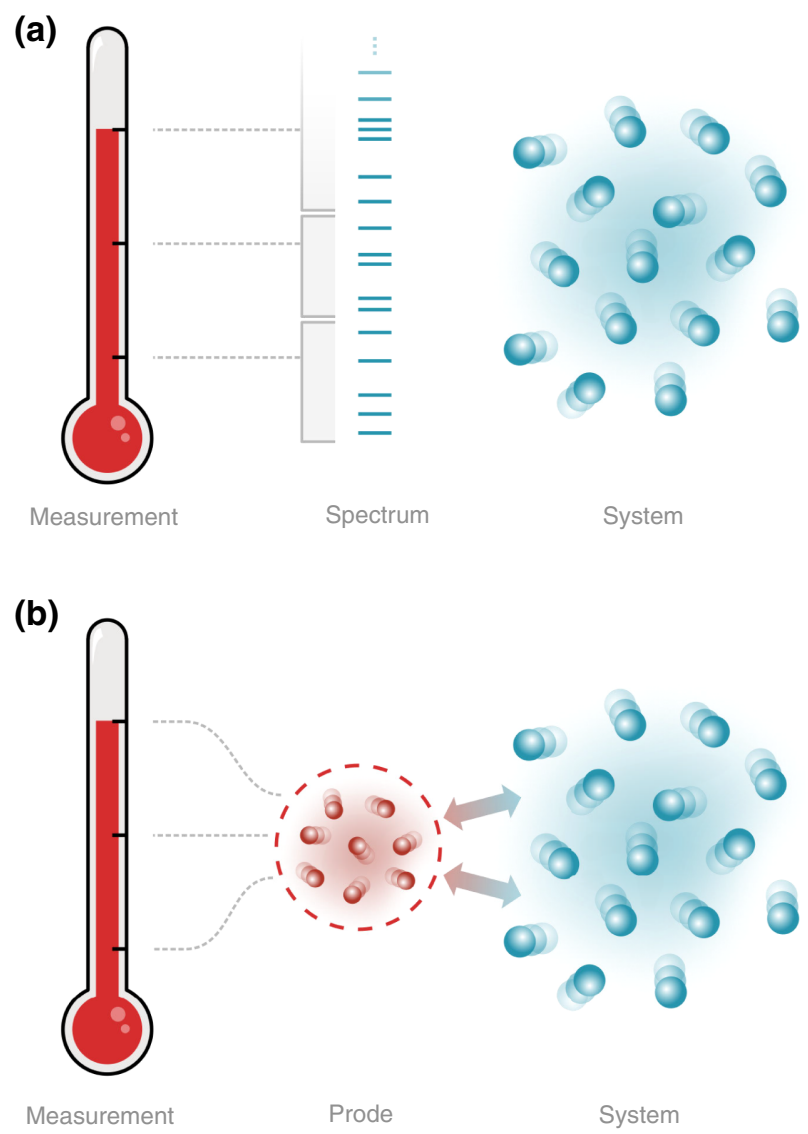

FIG. 1. (a) Thermometry with coarse-grained energy measurements. The measurement can be understood as resulting from postprocessing of a fine-grained, projective energy measurement. Energies are grouped into bins, and a single outcome is assigned to each bin. (b) Thermometry with probe-based measurements. A probe interacts unitarily with a target system. A measurement is then performed on the probe alone and used to infer the temperature of the target.

measuring a probe that has interacted with it [18,19,27,29,37] [see Fig. 1(b)]. All of these cases are examples of a coarse-grained measurement, which from an abstract point of view can be described by a $d$-outcome generalized quantum measurement of a $D$-dimensional system, with $d<D$. The fact that $d \ll D$ in most physically relevant cases may reduce the precision significantly. It is hence natural to ask what the optimal measurement strategy and the associated precision of temperature estimation are under such limitations.

In this paper, we develop a framework for addressing this question in detail. The framework is based on ideas from signal processing and parameter-estimation theory, and provides a simple, easy-to-use toolbox for studying coarse-grained thermometry of both few-body and manybody systems. We illustrate the framework by applying it to paradigmatic many-body models of spin lattices, both close to and far from criticality.
In the second part of the article, these abstract ideas are applied to probe-based temperature measurements. Here, the temperature of a sample is estimated by letting it interact with a probe (and possibly some auxiliary system) and then measuring the probe, as illustrated in Fig. 1(b). This type of measurements are particularly appealing since they provide a natural way to overcome one of the main challenges in thermometry: the design of noninvasive measurements, for example, for ultracold atomic gases [9,38-42]. In such probe-based measurements, a natural strategy is to let the probe reach thermal equilibrium with the sample [32]. However, it has been shown that the precision can be considerably enhanced by nonequilibrium strategies, where the probe interacts with the sample for a finite time $[9,22-28,43,44]$, couples strongly to the sample $[18,19]$, or uses an ancillary catalytic system [29]. Here we use our framework to obtain a fundamental bound on any such nonequilibrium strategy. We map the problem of probebased thermometry to that of coarse-grained thermometry, and determine the maximal sensitivity that can be obtained with a probe of dimension $d$. We construct a specific finetuned sample-probe interaction that always saturates the bound, and notably show that it can also be reached in relevant experimental situations. In particular, when the sample and the probe are described by a harmonic oscillator and a qubit, respectively, the optimal nonequilibrium estimation scheme in the low-temperature regime can be obtained via the Jaynes-Cummings Hamiltonian. This is of direct relevance to temperature measurements of BoseEinstein condensates [45] or micromechanical resonators $[22,23]$ via qubit probes.

\section{FRAMEWORK}

To be specific, we consider a system $S$ with a $D$ dimensional Hilbert space, described by a Hamiltonian $H$, and initially in a canonical thermal state

$$
\tau=\frac{1}{Z} e^{-\beta H},
$$

where $\beta=1 / T$ is the inverse temperature (we adopt units such that $\left.k_{B}=1\right)$ and $Z=\operatorname{Tr} e^{-\beta H}$ is the partition function. This is a family of states parameterized by the temperature, and the smallest variance in estimating this parameter, based on any measurement, is hence lower bounded by the quantum Cramér-Rao bound [46] (see also Refs. $[47,48])$

$$
\Delta T^{2} \geq \frac{1}{n \mathcal{F}(\tau)}
$$

where $n$ is the number of repetitions of the measurement and $\mathcal{F}$ is the quantum Fisher information with respect to $T$, which we refer to as the "thermal Fisher information." It is 
given by

$$
\mathcal{F}(\tau)=\beta^{4} \operatorname{var}(H)=\beta^{2} C,
$$

with $\operatorname{var}(H):=\left\langle H^{2}\right\rangle_{\tau}-\langle H\rangle_{\tau}^{2}$, where the angle brackets denote averaging over the quantum state $\left[\langle\hat{O}\rangle_{\tau}:=\right.$ $\operatorname{Tr}(\hat{O} \tau)]$, and where $C$ is the heat capacity of the system $(C:=d\langle H\rangle / d T)$. The optimal measurement, attaining the thermal Fisher information, is a projective measurement onto the eigenbasis of $H$ (i.e., a projective measurement of the system energy). In this optimal scenario, the more the energy fluctuates, the more precise the measurement can be in principle. The optimal measurement saturates the inequality (2) for arbitrary $n$ when the temperature estimator is unbiased. When an unbisaed estimator is not available, a large class of generic biased estimators will still asymptotically saturate the inequality (2) in the limit of many repetitions $(n \rightarrow \infty)$ [49]. Moreover, in the specific case of temperature estimation in many-body systems consisting of $N \gg 1$ particles, one can go even further and explicitly construct an estimator that, despite being biased for any finite $N$, will saturate the Cramér-Rao bound (2) as $N \rightarrow \infty$, even for $n=1$ [50].

However, when fine-grained measurements of energy are not available, while remaining valid, the bound (2) will in general become too loose. We formalize the problem as follows. Suppose the resolution of the experiment is limited to $d<D$ measurement outcomes. What is then the maximal precision for estimating the temperature of $S$ and which is the optimal $d$-outcome measurement achieving it? Below, we provide a systematic way to identify the optimal measurement.

Moreover, in Sec. V, we prove that the highest Fisher information achievable by a $d<D$ dimensional probe undergoing an arbitrary interaction with the sample is equal to the optimal $d$-outcome coarse-grained Fisher information in the above sense. We thus provide a fundamental benchmark for any conceivable protocol of probe-based thermometry. In particular, this includes any standard thermometric technique in current experimental setups.

\section{OPTIMAL COARSE-GRAINING}

We consider coarse-grained thermometry on a $D$ dimensional system. We take coarse-graining to mean that only generalized measurements, that is, positive operatorvalued measures (POVMs) with at most $d<D$ outcomes, are available. We then construct a framework for identifying the optimal POVM for thermometry in two steps.

First, we show that the optimal POVM is a projection onto energy subspaces of the system. This means that we can split the system spectrum into $d$ subsets and study measurements that project onto the corresponding eigensubspaces.
Second, we show that the optimal choice of subsets consists of consecutive "bins" (i.e., the sets are not interspersed). We then provide a method for constructing the optimal choice of bins for any given system spectrum. This is done by casting the problem in the language of an analogous signal-processing problem, known as Lloyd-Max quantization [51].

\section{A. Optimal POVM}

We take a $d$-outcome POVM $\mathcal{M}=\left\{M_{\alpha}\right\}_{\alpha=1}^{d}$ and a system in the thermal state $\tau$ of Eq. (1). Each outcome $\alpha$ then occurs with probability

$$
p_{\alpha}=\operatorname{Tr}\left(M_{\alpha} \tau\right)
$$

This distribution contains information about the temperature $T$, as quantified by the Fisher information [52]

$$
\mathcal{C}=\sum_{\alpha=1}^{d} \frac{1}{p_{\alpha}}\left(\frac{\partial p_{\alpha}}{\partial T}\right)^{2},
$$

which for a thermal state becomes [30]

$$
\mathcal{C}=\beta^{4} \sum_{\alpha} \frac{1}{p_{\alpha}}\left\{\operatorname{Tr}\left[\tau M_{\alpha}\left(H-\langle H\rangle_{\tau}\right)\right]\right\}^{2}
$$

Note that, after coarse-graining, $p_{\alpha}$ is no longer in the socalled exponential family with respect to $T$ [53], which means that no temperature estimator can satisfy the Cramér-Rao bound for any finite number of repetitions $n$ [49]. However, the Fisher information $(\mathcal{C})$ is still a key precision quantifier, due to the fact that, for all unbiased and certain generic biased estimators, such as the maximum likelihood estimator, the Cramer-Rao bound is saturated asymptotically in the $n \rightarrow \infty$ limit. Moreover, in the same limit, the Fisher information retains its key role even in the Bayesian estimation approach [54].

We thus set as our goal to determine the optimal $d$ outcome POVM that maximizes the Fisher information $\mathcal{C}$.

We first observe that the POVM elements $M_{\alpha}$ can be taken to be diagonal in the energy eigenbasis. Because $\tau$ is diagonal in this basis, only diagonal elements of $M_{\alpha}$ contribute to the probability $p_{\alpha}$ in Eq. (4). Dropping all offdiagonal elements from each $M_{\alpha}$ results in a valid POVM since the operators remain positive and still sum to identity. Hence, for every POVM there exists a diagonal POVM that achieves the same $p_{\alpha}$ and thus the same Fisher information. It is therefore sufficient to consider diagonal POVMs when one is looking for an optimal POVM maximizing $\mathcal{C}$.

Next, we note that $\mathcal{C}$ is convex with respect to the POVM. That is, denoting by $\mathcal{C}_{\mathcal{M}}$ the Fisher information corresponding to a particular POVM and considering two 
POVMs $\mathcal{M}$ and $\mathcal{N}$ and a mixing parameter $0 \leq \lambda \leq 1$, we have

$$
\mathcal{C}_{\lambda \mathcal{M}+\bar{\lambda} \mathcal{N}} \leq \lambda \mathcal{C}_{\mathcal{M}}+\bar{\lambda} \mathcal{C}_{\mathcal{N}}
$$

where $\bar{\lambda}=1-\lambda$. This can be seen by rewriting Eq. (6) as

$$
\mathcal{C}=\beta^{4} \sum_{\alpha} \frac{1}{p_{\alpha}} W_{\alpha}^{2}
$$

where $W_{\alpha}=\operatorname{Tr}\left[\tau M_{\alpha}\left(H-\langle H\rangle_{\tau}\right)\right]$. Both $p_{\alpha}$ and $W_{\alpha}$ are linear in the POVM operators. Hence, when mixing POVMs, $W_{\alpha}^{(\lambda \mathcal{M}+\bar{\lambda} \mathcal{N})}=\lambda W_{\alpha}^{(\mathcal{M})}+\bar{\lambda} W_{\alpha}^{(\mathcal{N})}$ and $p_{\alpha}^{(\lambda \mathcal{M}+\bar{\lambda} \mathcal{N})}=$ $\lambda p_{\alpha}^{(\mathcal{M})}+\bar{\lambda} p_{\alpha}^{(\mathcal{N})}$. Equation (7) is then an immediate consequence of the fact that $(1 / p) W^{2}$ is a jointly convex function of $p$ and $W$ (see, e.g., Ref. [55]).

Finally, we show that the optimal POVM is necessarily a collection of nonoverlapping projectors onto eigensubspaces of $H$. Indeed, take a POVM $\mathcal{M}$ such that for some eigenstate $|k\rangle$ of $H$ there are at least two POVM elements for which $\left\langle k\left|M_{\alpha}\right| k\right\rangle>0$. Define

$$
\varsigma_{\alpha}=\left\langle k\left|M_{\alpha}\right| k\right\rangle .
$$

Now construct $d$ new POVMs $\mathcal{N}^{(\gamma)}$, with elements

$$
N_{\alpha}^{(\gamma)}=M_{\alpha}+\left(\delta_{\alpha \gamma}-\varsigma_{\alpha}\right)|k\rangle\langle k| .
$$

Each $\mathcal{N}^{(\gamma)}$ has the property that only $N_{\gamma}^{(\gamma)}$ has a nonzero $k$ th diagonal; namely, $\left\langle k\left|N_{\gamma}^{(\gamma)}\right| k\right\rangle=1$ while $\left\langle k\left|N_{\alpha \neq \gamma}^{(\gamma)}\right| k\right\rangle=$ 0 . Furthermore, we note that

$$
\sum_{\gamma} \varsigma_{\gamma} \mathcal{N}^{(\gamma)}=\mathcal{M}
$$

Since the $M_{\alpha}$ form a POVM, we have $\varsigma_{\alpha} \geq 0$ and $\sum_{\alpha} \varsigma_{\alpha}=$ 1 . By the convexity (7) of the Fisher information,

$$
\mathcal{C}_{\mathcal{M}} \leq \sum_{\gamma} \varsigma_{\gamma} \mathcal{C}_{\mathcal{N}(\gamma)}
$$

and it follows that there must be at least one $\gamma$ for which $\mathcal{C}_{\mathcal{N}^{(\gamma)}}(T) \geq \mathcal{C}_{\mathcal{M}}(T)$. This means that the optimal POVM will have to be one that consists of nonoverlapping projectors on eigensubspaces of $H$. Given a (possibly degenerate) Hamiltonian $H=\sum_{i} E_{i}|i\rangle\langle i|$ the optimal POVM will thus be of the form

$$
\Pi_{\alpha}=\sum_{E_{i} \in I_{\alpha}}|i\rangle\langle i|
$$

where the $I_{\alpha}$ define a partition of the set of all eigenenergies into nonoverlapping subsets ("bins").

To summarize, optimal, coarse-grained thermometry can always be achieved by considering projective measurements onto nonoverlapping eigenenergy subspaces.

\section{B. Optimal binning}

We now construct a method for determining the optimal eigenenergy subsets defining the optimal POVM.

For convenience, we choose the basis of $H$ such that

$$
E_{1} \leq E_{2} \leq \cdots \leq E_{D}
$$

and write the probability of finding the system in bin $I_{\alpha}$ as [cf. Eq. (4)]

$$
p_{\alpha}=\operatorname{Tr}\left(\Pi_{\alpha} \tau\right)=\sum_{E_{i} \in I_{\alpha}} q_{i},
$$

where $q_{i}=\exp \left(-\beta E_{i}\right) / Z$. Next we introduce the "bin energies" (normalized average energy within each bin),

$$
\epsilon_{\alpha}=\frac{1}{p_{\alpha}} \sum_{E_{i} \in I_{\alpha}} q_{i} E_{i} .
$$

With these definitions, we re-express the Fisher information in Eq. (6) for the corresponding measurement as

$$
\mathcal{C}=\beta^{4} \sum_{\alpha=1}^{d} p_{\alpha}\left(\epsilon_{\alpha}-\langle H\rangle\right)^{2} .
$$

We henceforth refer to this as the "coarse-grained Fisher information." Compared with Eq. (3), the expression for $\mathcal{C}$ describes how each of the energies $\epsilon_{\alpha}$ fluctuates away from the average.

As a first step toward finding the optimal sets $I_{\alpha}$, in Appendix A, applying a result from Ref. [56], we prove that the best choice of $I_{\alpha}$ is given by a binning into consecutive intervals: $I_{\alpha}=\left\{E_{i_{\alpha-1}}, E_{i_{\alpha-1}+1}, \ldots, E_{i_{\alpha-1}+\left|I_{\alpha}\right|-1}\right\}$, where $1 \leq i_{\alpha-1} \leq D$ and the iteration rule is $i_{\alpha}=i_{\alpha-1}+$ $\left|I_{\alpha}\right|$. Introducing the "boundaries" $b_{\alpha}:=E_{i_{\alpha}}$, we can conveniently write $I_{\alpha}=\left[b_{\alpha-1}, b_{\alpha}\right)$, with the proviso that $b_{0}=$ $E_{1}$ and $b_{d}=E_{D+1}=E_{D}$ (the extra level $E_{D+1}$ will not enter into any physical quantity; it is introduced just so that $E_{D}$ enters $I_{d}$ despite $\left[b_{d-1}, b_{d}\right)$ having an open end). Note that, for discrete spectra, the boundaries $b_{\alpha}$ need not be exactly at energy eigenvalues. Positioning a boundary anywhere between neighboring eigenvalues results in the same POVM in Eq. (13).

The remaining task is then to find the optimal intervals $I_{\alpha}=\left[b_{\alpha-1}, b_{\alpha}\right)$ that maximize $\mathcal{C}$. This will give the best strategy for temperature estimation using a $d$-outcome POVM.

Before carrying out this optimization, it is useful to recast the problem in terms of the density of states (DOS)

$$
\Omega(E)=\sum_{i} \delta\left(E-E_{i}\right),
$$

where $\delta$ denotes the Dirac $\delta$ function (note that this definition does not assume a continuous spectrum). Expectation values of any function $g(H)$ of the Hamiltonian may 
then be written as

$$
\langle g(H)\rangle=\sum_{i} g\left(E_{i}\right) q_{i}=\int d E g(E) \Omega(E) \frac{e^{-\beta E}}{Z} .
$$

This means that we can define the distribution of energy as

$$
q(E)=\Omega(E) \frac{e^{-\beta E}}{Z}
$$

so expectation values can be computed simply in terms of integrals over $q(E)$. This way, the probabilities $p_{\alpha}$ in Eq. (15) and the bin energies in Eq. (16) can be conveniently written as

$$
p_{\alpha}=\int_{b_{\alpha-1}}^{b_{\alpha}} d E q(E) \quad \text { and } \quad \epsilon_{\alpha}=\frac{1}{p_{\alpha}} \int_{b_{\alpha-1}}^{b_{\alpha}} d E q(E) E .
$$

These quantities are therefore all expressed explicitly as functions of the boundaries $b_{\alpha}$.

The advantage of introducing the DOS is twofold. First, it allows for a unified treatment of Hamiltonians with discrete and quasicontinuous spectra (as one would expect in quantum many-body systems). Second, it allows us to frame the problem in the language of signal processing. A common task in signal processing is to transmit a continuous function $q(E)$ through a channel. This is usually done by discretizing the function into a finite set of bins $I_{\alpha}=\left[b_{\alpha-1}, b_{\alpha}\right)$. The question is then which choice of bins leads to the optimal transmission. This problem is solved by using the scheme known as Lloyd-Max quantization (see Chap. 3 in Ref. [51]). If one uses the mean squared variations of energy as a figure of merit, one sees that the maximization of the Fisher information [see Eq. (17)] becomes entirely analogous to this signal-processing problem.

To proceed, we introduce the quantity

$$
\mathcal{D}=\beta^{4} \sum_{\alpha=1}^{d} \int_{b_{\alpha-1}}^{b_{\alpha}} d E q(E)\left(E-\epsilon_{\alpha}\right)^{2}
$$

It can be directly verified that the thermal Fisher information given by Eq. (3) can be decomposed as

$$
\mathcal{F}=\mathcal{C}+\mathcal{D}
$$

which means that the task of maximizing $\mathcal{C}$, for a fixed $\mathcal{F}$, is tantamount to that of minimizing $\mathcal{D}$.

The minimization can be done in the usual way, by equating to zero the derivatives of $\mathcal{D}$ with respect to $b_{\alpha}$. A straightforward calculation shows that the minima of
$\mathcal{D}$ occur when the intervals $b_{\alpha}$ satisfy the implicit (and generally nonlinear) equations [57]

$$
b_{\alpha}=\frac{\epsilon_{\alpha+1}+\epsilon_{\alpha}}{2}, \quad \alpha=1, \ldots, d-1 .
$$

These equations are implicit because $\epsilon_{\alpha}$ itself is a function of the $\left\{b_{\alpha}\right\}$ [Eq. (16)]. This summarizes the core of our framework. Equation (24) provides a recipe for how to optimize the energy bins in a $d$-outcome POVM in order to maximize the thermometric precision.

\section{Illustrative examples}

We now present two examples using our framework for optimal coarse-grained thermometry.

\section{Noninteracting qubits}

A simple but illustrative example is a system of $N$ identical, noninteracting qubits. The system is in a thermal state, and we take the ground-state and excited-state energies to be 0 and 1 , respectively. The energy levels of the system will thus range from 0 to $N$ in integer steps. The probability of finding the system in a state with energy $j$ is then

$$
q_{j}=\left(\begin{array}{c}
N \\
j
\end{array}\right) s^{j} r^{N-j},
$$

where we have defined the excited-state population $s=$ $1 /\left(e^{\beta}+1\right)$ and $r=1-s$. For a $d$-outcome measurement, the probabilities and bin energies take the forms

$$
\begin{aligned}
p_{\alpha} & =\sum_{j=b_{\alpha-1}}^{b_{\alpha}-1}\left(\begin{array}{l}
N \\
j
\end{array}\right) s^{j} r^{N-j}, \\
\epsilon_{\alpha} & =\frac{1}{p_{\alpha}} \sum_{j=b_{\alpha-1}}^{b_{\alpha}-1}\left(\begin{array}{l}
N \\
j
\end{array}\right) j s^{j} r^{N-j},
\end{aligned}
$$

where the bin positions $b_{\alpha}$, which are integers in this case, are determined from Eq. (24) (with $b_{0}=0$ ). For this system, the average energy is simply $\langle H\rangle=N s$, while the thermal Fisher information (3) is $\mathcal{F}=\beta^{4} N r s$. In Fig. 2, we show the ratio $\mathcal{C} / \mathcal{F}$ as a function of the bin positions $b_{\alpha}$ for the cases $d=2,3$. The bins $b_{\alpha}$ that maximize $\mathcal{C} / \mathcal{F}$ are precisely the solutions of Eq. (24).

According to the de Moivre-Laplace theorem, in the $N \gg 1$ limit, the distribution $q_{j}$ becomes Gaussian. Using this, we show in Appendix B that for binary measurements $(d=2)$ and $N \gg 1$, the optimal binning strategy leads to

$$
\mathcal{C}=\frac{2}{\pi} \mathcal{F}
$$

and is achieved when the boundary is inserted at $b=$ $\langle H\rangle=N s$. This result is noteworthy; it shows that irrespective of the number of qubits $N$, it is always possible to 

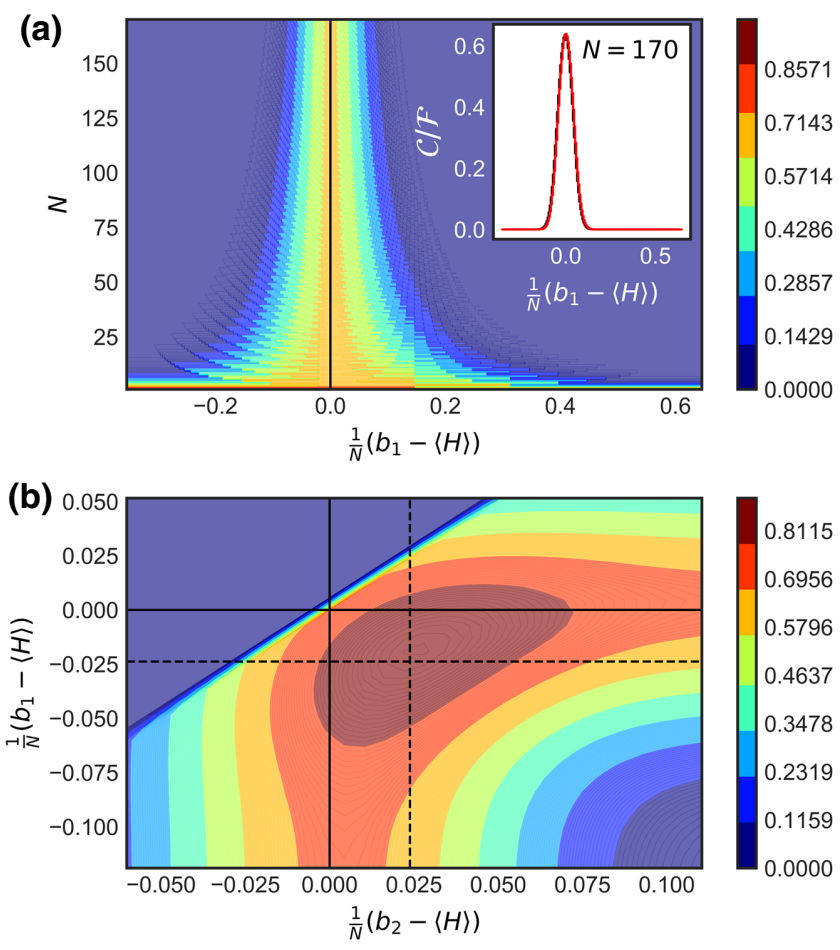

FIG. 2. Ratio $\mathcal{C} / \mathcal{F}$ between the coarse-grained Fisher information and the thermal Fisher information at $\beta=0.6$. (a) The case of $d=2$, plotted as a function of the partition $\left(b_{1}-\langle H\rangle\right) / N$ and the number of qubits $N$. The ratio reaches a maximal value of approximately 0.64 in the large- $N$ limit. The inset shows the cross section at $N=170$. (b) The case of $d=3$ for $N=170$, plotted as a function of the lower partition $\left(b_{1}-\langle H\rangle\right) / N$ and the upper partition $\left(b_{2}-\langle H\rangle\right) / N$. The ratio has a maximal value of approximately 0.82 . The dashed lines gives the optimal partitions as predicted by a symmetric partition around the mean energy.

construct a dichotomic measurement strategy that captures $2 / \pi \approx 0.63$ of the full thermal Fisher information.

\section{Linear density of states}

As a contrast with the $N$-qubit case, we now consider an example of a system with a continuous spectrum. Namely, we assume the system has a linear density of states: $\Omega(E) \propto E$. Such a DOS is met, for instance, in a noninteracting, ultrarelativistic gas in two dimensions. If the system is in a thermal state [Eq. (1)], the average energy is simply $\langle H\rangle=2 / \beta$ and the variance is $\operatorname{var}(H)=2 / \beta^{2}$. Thus, the thermal Fisher information given in Eq. (3) is $\mathcal{F}=2 \beta^{2}$.

We first consider the case of binary measurements, $d=$ 2 , defined by a single boundary $b$. The probabilities $p_{1}$ and $p_{2}$ [Eq. (21)] are then given by

$$
p_{1}=1-p_{2}, \quad p_{2}=\int_{b}^{\infty} d E q(E)=(1+\beta b) e^{-\beta b},
$$

and the corresponding bin energies become

$$
\epsilon_{1}=\frac{2}{\beta}+\frac{\beta b^{2}}{1+b \beta-e^{b \beta}}, \quad \epsilon_{2}=\frac{2}{\beta}+\frac{\beta b^{2}}{1+\beta b} .
$$

Thus, the Fisher information for the measurement, Eq. (17), is

$$
\mathcal{C}=\frac{\beta^{6} b^{4}}{(1+\beta b)\left(e^{\beta b}-1-\beta b\right)} .
$$

To find the optimal partition, we solve Eq. (24) for $b$; that is, $b=\left(\epsilon_{1}+\epsilon_{2}\right) / 2$. This is a nonlinear equation that can be solved numerically. A plot of $\mathcal{C} / \mathcal{F}$ is shown in the inset in Fig. 3(a). It attains a maximum at $\beta b \approx 2.589$. At this point $\mathcal{C} \approx 0.643 \mathcal{F}$; that is, the binary measurement reaches approximately $64 \%$ of the maximal Fisher information for any possible measurement [this is slightly higher than in Eq. (27)].

The dependence of $\mathcal{C}$ on the number of outcomes $d$, for optimal binnings, is shown in Fig. 3(a). Quite remarkably, even with as few as eight bins, one can already reach a precision of approximately $97 \%$ of $\mathcal{F}$ - the maximal possible precision. An illustration of the probabilities $p_{k}$ and the corresponding bins $b_{k}$ is given in Fig. 3(b) for $d=8$.

\section{General remarks and extension to imperfect measurements}

The two examples in Sec. III C carry an important general message: even measurements so coarse-grained as to have only two outcomes can estimate the temperature of a generic system with precision (as quantified by the Fisher information) that is proportional to the ultimate precision - the thermal Fisher information [Eq. (3)]. In Appendix $\mathrm{C}$, we show that this is not a coincidence by proving that any system for which the energy distribution is unimodal and has sufficiently fast decaying tails displays a proportionality $\mathcal{C} \propto \mathcal{F}$. More specifically, we prove that there exists a finite number $\Xi \in[0,1]$ such that $\mathcal{C} \geq \Xi \mathcal{F}$.

As will be discussed in Sec. IV, the relevance of these results lies in the fact that unimodal energy distributions with quickly decaying tails are a generic behavior expected in finite-temperature many-body systems with short-range interactions, both at and away from criticality. In fact, we will show that $\Xi=2 / \pi$, as in Eq. (27), actually happens for a large variety of interacting lattice models. It is of course possible to conceive nonunimodal energy distributions for which the proportionality $\mathcal{C} \propto \mathcal{F}$ breaks down. We illustrate this in Appendix D, where we construct an example for which $\mathcal{C} / \mathcal{F} \rightarrow 0$ when $N \rightarrow \infty$.

Our framework can also be adapted to scenarios where the ideal energy binning cannot be implemented and imprecisions are present. In the simplest case, one could have some imprecision in determining the optimal bins through Eq. (24), which would lead to a decrease in $\mathcal{C}$. For 

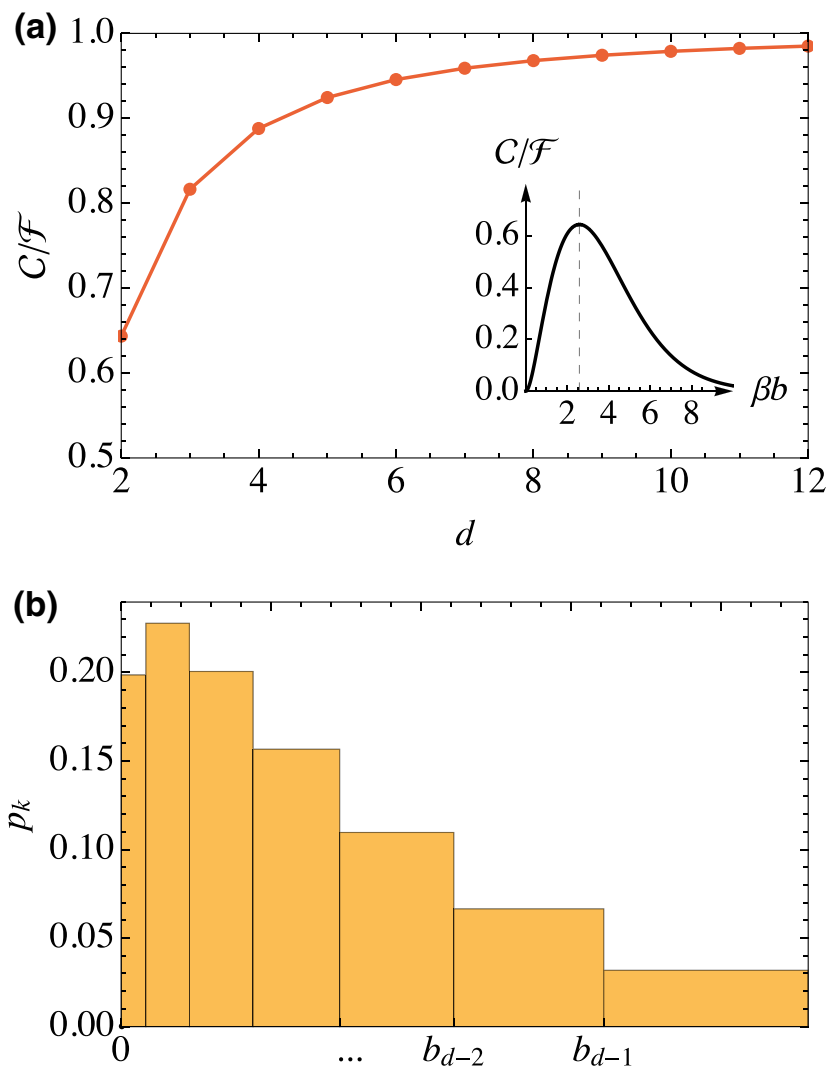

FIG. 3. Optimal thermometry binning for a system described by a linear density of states: $\Omega(E) \propto E$. (a) Optimal binned Fisher information, Eq. (17), as a function of $d$. The curves are normalized by the full Fisher information, which in this case reads $\mathcal{F}=2 \beta^{2}$. The inset shows the ratio $\mathcal{C} / \mathcal{F}$ as a function of the binning position $b$ for $d=2$. The optimal bin occurs at $\beta b=2.58975$. The points in the main plot are already optimized over the binning positions. (b) The corresponding probabilities $p_{k}$ and average bins $b_{k}$ for $d=8$.

the case $d=2$, this is illustrated in the inset in Fig. 3(a), which shows how $\mathcal{C} / \mathcal{F}$ decreases as the bin position deviates from its optimal value.

Another way in which imprecisions can enter our framework is when the POVMs (4) are noisy. For instance, experimental errors may cause energies close to a bin edge to sometimes result in outcomes corresponding to neighboring bins. Such effects can be accounted for within our framework by modifying Eq. (21). To see that, we first rewrite Eq. (21) as

$$
p_{\alpha}=\int_{-\infty}^{\infty} d E q(E) B_{\alpha}(E)
$$

and similarly for $\epsilon_{\alpha}$. Here $B_{\alpha}(E)$ is a boxcar function, with value 1 when $E \in I_{\alpha}=\left[b_{\alpha-1}, b_{\alpha}\right)$ and 0 otherwise. A similar analysis can also be done for the discrete representation of Eq. (15). In this case, we would have $p_{\alpha}=\sum_{i} B_{\alpha, i} q_{i}$, where $B_{\alpha, i}$ is a matrix with entries 1 when $E_{i} \in I_{\alpha}$ and 0
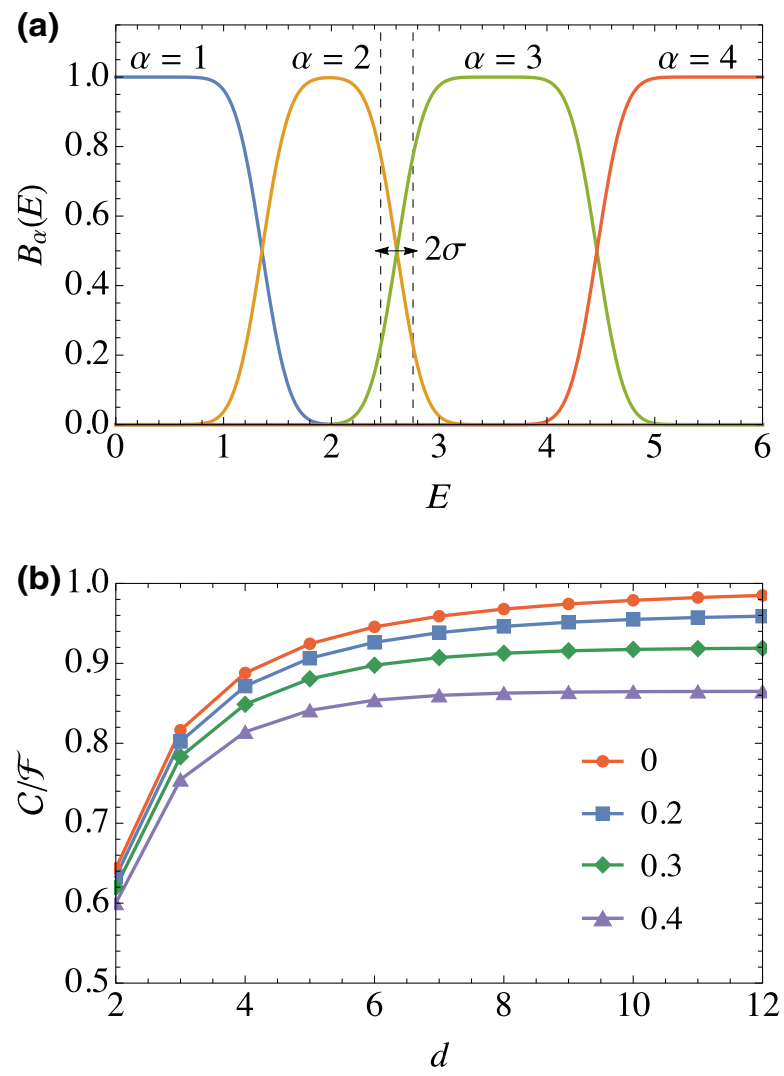

FIG. 4. The role of imperfect measurements in optimal thermometry. (a) Example of smoothed boxcars, Eq. (30), for $\sigma=$ 0.2. (b) Worsening of the coarse-grained Fisher information $\mathcal{C}$ for the linear DOS example studied in Sec. III C 2. The red curve is the same as in Fig. 3(a), while the other curves are computed using the smoothed boxcars (30) with $\sigma=0.2,0.3$, and 0.4 (in the unit of $\beta=1$ ).

otherwise. However, it is more convenient to work with the continuous-energy representation (29).

It is now straightforward to generalize Eq. (29) to include the effects of noise by replacing $B_{\alpha}(E)$ by a different function; for instance, a smoothed boxcar as depicted in Fig. 4(a). Since $\sum_{\alpha} p_{\alpha}=1$ for any initial distribution $q(E)$, it follows that $\sum_{\alpha} B_{\alpha}(E)=1$ for all $E$. This can be viewed as a normalization condition for $B_{\alpha}(E) . B_{\alpha}(E)$ is actually a combination of a stochastic matrix (whose columns add up to 1) and an isometry, which reduces the dimension from a continuous energy $E$ to a discrete set of outcomes $\alpha$. The precise form of $B_{\alpha}(E)$ will depend on the details of the experiment.

Measurement errors can cause not only a loss of precision but also systematic shifts in energy by, for example, falsely displacing the energies $\epsilon_{\alpha}$ by a certain amount. For simplicity, we study these kinds of imprecision separately. We defer the discussion of robustness to energy shifts to Secs. IV A and IV B, while here we choose $B_{\alpha}(E)$ to be symmetric in the interval $\left[b_{\alpha-1}, b_{\alpha}\right)$ and centered at the 
midpoint $\left(b_{\alpha-1}+b_{\alpha}\right) / 2$ so that the bin energies $\epsilon_{\alpha}$ are not displaced.

The remaining feature to be described is errors associated with imperfect binning. This can again be done with the smoothed boxcar of Fig. 4(a):

$$
B_{\alpha}(E)=\frac{1}{2} \operatorname{erf}\left(\frac{b_{\alpha}-E}{\sigma \sqrt{2}}\right)-\frac{1}{2} \operatorname{erf}\left(\frac{b_{\alpha-1}-E}{\sigma \sqrt{2}}\right),
$$

where erf $x$ is the error function and $\sigma$ is a parameter measuring the degree of imprecision (a sharp boxcar is recovered when $\sigma \rightarrow 0$ ). A function of this form defines a certain energy window $2 \sigma$, where measurements associated to one bin can be recorded in another bin. For this reason, wider bins tend to be less affected than thinner ones, which is physically reasonable.

We illustrate the above ideas with the linear DOS example from Sec. III C2. In Fig. 4(b) we present $\mathcal{C} / \mathcal{F}$ as a function of $d$ for binning strategies computed with the smoothed boxcars (30), with different values of $\sigma$. This is contrasted with the ideal case, shown in red, which coincides with the red curve in Fig. 3(a). As can be seen, unsharp bin edges necessarily decrease the coarse-grained Fisher information. That said, $\mathcal{C} / \mathcal{F}$ is surprisingly robust: even when the smearing occurs over a large part of the bin width (e.g., $30 \%), \mathcal{C} / \mathcal{F}$ does not decrease much (only about $7 \%)$.

\section{MANY-BODY LATTICE MODELS}

We now proceed to analyze quantum systems on a lattice, which is one of the most physically relevant settings where coarse-grained measurements could be useful. We start with general considerations and a tight-binding chain as an illustrative example. Then we show a general result for all noncritical spin models, and conclude with an analysis of a system undergoing a thermal phase transition.

\section{A. Gaussian density of states}

In many-body lattice models, the energy distribution (20) often displays an approximate Gaussian form in the thermodynamic limit [58-60] (see also the detailed discussion in Sec. IV B and Appendix E). As a simple, illustrative example of this principle, consider a fermionic one-dimensional (1D) tight-binding chain with $N$ sites under periodic boundary conditions:

$$
H=\sum_{k=1}^{N} \varepsilon \hat{c}_{k}^{\dagger} \hat{c}_{k}-t \sum_{k=1}^{N}\left(\hat{c}_{k+1}^{\dagger} \hat{c}_{k}+\hat{c}_{k}^{\dagger} \hat{c}_{k+1}\right),
$$

where $\hat{c}_{k}$ is the fermionic annihilation operator at site $k, \varepsilon$ is the on-site energy, $t$ is the hopping (tunneling) strength, and $\hat{c}_{k+N}=\hat{c}_{k}$ ensures periodic boundary conditions. When diagonalized, the Hamiltonian of this model
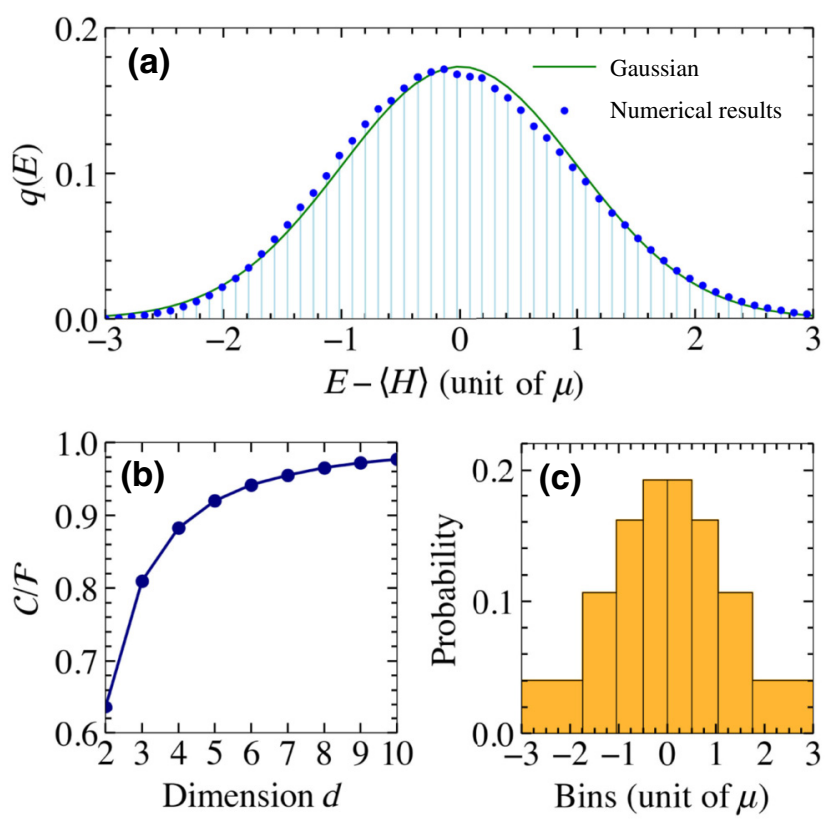

FIG. 5. (a) Energy distribution for a fermionic tight-binding chain with 20 sites. The hopping strength is $t=0.3$ and the temperature is $T / \epsilon=2$. The numerical results are compared with the Gaussian distribution [Eq. (33)]. (b) Optimal binned Fisher information for the general Gaussian distribution as a function of $d$. The curves are normalized by the thermal Fisher information [Eq. (3)]. (c) Optimal binning and the corresponding probabilities for the general Gaussian distribution with $d=8$.

takes the form $[30,61]$

$$
H=\sum_{a=1}^{N} \varepsilon_{a} \hat{C}_{a}^{\dagger} \hat{C}_{a}
$$

with the (linearly) transformed $\hat{C}_{a}$ 's satisfying standard fermionic anticommutation relations, and with eigenenergies given by $\varepsilon_{a}=\varepsilon-t \cos (2 \pi a / N)$.

We numerically compute the energy distribution (20) of this model and in Fig. 5(a) compare the results with a continuous Gaussian distribution with average energy $\langle H\rangle$ and variance $\mu^{2} \equiv \operatorname{var}(H)=-\partial\langle H\rangle / \partial \beta$ (both of which depend implicitly on $T$ ); that is,

$$
q(E)=\frac{1}{\sqrt{2 \pi \mu^{2}}} e^{-(E-\langle H\rangle)^{2} /\left(2 \mu^{2}\right)} .
$$

We observe that the Gaussian distribution is a good approximation already with a modest number of sites and a modest hopping strength. The approximation improves with the number of sites and becomes exact in the thermodynamic limit (see Refs. [59,60]).

We now take a Gaussian distribution as a given and compute the Fisher information for different coarse-grainings 
of the continuous distribution (33). In this case, the probabilities and bin energies in Eq. (21) become

$$
\begin{aligned}
p_{\alpha} & =\frac{1}{2}\left(\operatorname{erf} \tilde{b}_{\alpha}-\operatorname{erf} \tilde{b}_{\alpha-1}\right), \\
\epsilon_{\alpha} & =\langle H\rangle-\frac{\mu}{p_{\alpha} \sqrt{2 \pi}}\left(e^{-\tilde{b}_{\alpha}^{2}}-e^{-\tilde{b}_{\alpha-1}^{2}}\right),
\end{aligned}
$$

where $\tilde{b}_{\alpha}:=\left(b_{\alpha}-\langle H\rangle\right) /(\mu \sqrt{2})$ (with $b_{0}=-\infty$ and $b_{d}=$ $\infty)$ are the shifted and rescaled bin positions. The full Fisher information is simply $\mathcal{F}=\beta^{4} \mu^{2}$. For a $d$-outcome measurement, one can then numerically perform the optimization according to Eq. (24) to find the best such measurement and the corresponding coarse-grained Fisher information (17). The results for $\mathcal{C} / \mathcal{F}$ for different numbers of bins $d$ are shown in Fig. 5(b). As in the linear density of states case, one sees a quick growth of $\mathcal{C}$ with $d$ toward the maximal value $\mathcal{F}$.

The particular case of $d=2$ can be obtained by setting $b_{0}=-\infty, b_{2}=\infty$, and $b_{1}=b$. We then get

$$
p_{1,2}=\frac{1 \pm \operatorname{erf} \tilde{b}}{2}, \quad \epsilon_{1,2}=\langle H\rangle \mp \frac{\mu}{\sqrt{2 \pi} p_{1,2}} e^{-\tilde{b}^{2}},
$$

so the coarse-grained Fisher information [Eq. (17)] becomes

$$
\mathcal{C}=\frac{2 \mathcal{F}}{\pi} \frac{e^{-2 \tilde{b}^{2}}}{1-(\operatorname{erf} \tilde{b})^{2}} .
$$

The result is expressed solely in terms of the shifted bin position $\tilde{b}$; therefore, the minimization procedure is independent of $\langle H\rangle$ or $\mu$. As one may readily verify, the function in Eq. (35) is maximized at $\tilde{b}=0$. That is, the bin should be placed symmetrically, at $b=\langle H\rangle$. The corresponding maximum is

$$
\mathcal{C}=\frac{2}{\pi} \mathcal{F}
$$

This relation is robust with respect to imprecise identification of the optimal boundary (which can be understood as a systematic error in the energy measurement, as mentioned in Sec. IIID). Indeed, Taylor-expanding the right-hand side of Eq. (35) with respect to small $\tilde{b}$ around its optimal value, $\tilde{b}=0$, we find that $\mathcal{C} / \mathcal{F}=(2 / \pi)\left[1-2 \tilde{b}^{2}(1-\right.$ $\left.2 / \pi)+O\left(\tilde{b}^{4}\right)\right]$. Even for a significant deviation of $\mid b-$ $\langle H\rangle \mid=0.3 \mu, \mathcal{C} / \mathcal{F}$ degrades by only approximately $3.3 \%$.

Not coincidentally, the relation in Eq. (36) also appears in the case of noninteracting qubits in the limit of large $N$ (Sec. III C 1). This is because the energy distribution in that case also becomes Gaussian in the $N \gg 1$ limit, due to the central limit theorem.

In Fig. 5(c), we illustrate the optimal bins and the corresponding probabilities for the distribution (33) in the case of $d=8$. In this case, the optimal bins have to be located numerically. Unsurprisingly, it is found that the optimum is symmetric around the average energy.

\section{B. Noncritical, interacting systems on lattices}

We will now show how some of the conclusions of Sec. IV A actually hold universally in the thermodynamic limit. Intuitively speaking, the idea is that generic lattice models with finite-range interactions, when away from criticality, tend to have a Gaussian energy distribution due to the many-body Berry-Esseen theorem [58-60]. Therefore, the same behavior as in Fig. 5 is expected to occur when coarse-graining to different partitions in such lattice models.

In Appendix $\mathrm{E}$, we prove that the maximal $\mathcal{C} / \mathcal{F}$ achievable by two-outcome measurements $(d=2)$ is $\frac{2}{\pi}+$ $O\left(\ln ^{-1} N\right)$, and the boundary of the optimal partition $I_{1}=$ $\left[E_{0}, b\right]$ and $I_{2}=\left(b, E_{D}\right]$ is near the average energy: $b-$ $\langle H\rangle=O\left(\ln ^{-1 / 2} N\right) \sqrt{\operatorname{var}(H)}$. Moreover, when $b=\langle H\rangle$ exactly, one still has $\frac{\mathcal{C}}{\mathcal{F}}=\frac{2}{\pi}+O\left(\ln ^{-1} N\right)$. In the thermodynamic limit, this coincides with the results for the exact Gaussian distribution (Sec. IV A) and for independent qubits (Sec. III C 1). We expect that for $d \geq 3$ partitions, one should be able to prove results identical to those obtained for the exact Gaussian energy distribution in Sec. IV A by using arguments along the lines of those in Appendix E.

To prove Eq. (36), we need to assume that the thermal state of the lattice satisfies the following two generic conditions:

(i) Exponential decay of correlations: For arbitrary regions $\mathcal{X}, \mathcal{Y}$ separated by a distance $l$ on the lattice, and some constant $\xi$,

$$
\max _{X \in \mathcal{X}, Y \in \mathcal{Y}}\left|\frac{\langle X \otimes Y\rangle-\langle X\rangle\langle Y\rangle}{\|X\|\|Y\|}\right| \leq e^{-l / \xi} .
$$

(ii) The variance in energy scales linearly with the number of lattice sites: $\operatorname{var}(H)=\left\langle H^{2}\right\rangle-\langle H\rangle^{2}=s^{2} N$.

Assumption (i) is expected to hold for a very large class of systems away from criticality. Indeed, it has been rigorously proven for $1 \mathrm{D}$ translation-invariant thermal states [62], finite-range fermionic lattice systems of arbitrary spatial dimension at nonzero temperatures [63], and all finiterange lattice systems above a threshold lattice-dependent temperature [64]. Assumption (ii) is expected to hold for most systems at a high enough temperature. Note that assumption (i) already implies that $\operatorname{var}(H)=O(N)$ [65].

The detailed proof of Eq. (36) in Appendix E is based on the Berry-Esseen theorem for local Hamiltonians that relies on the two assumptions above and is proven in Refs. $[59,60]$ (see also Appendix F 1). This result can be seen as a strengthening of the central limit theorem, which gives 
a precise notion of how the energy distribution of lattice models converges to a Gaussian in the thermodynamic limit. It allows us to estimate functions of the form of Eq. (19), in this case, the bin energies $\epsilon_{k}$.

Lastly, the Gaussian behavior of noncritical many-body lattice systems also extends to the problem of how robust $\mathcal{C} / \mathcal{F}=2 / \pi$ is with respect to imprecise identification of the optimal binning boundary. Indeed, as we show in Appendix E, for small $\tilde{b}=(b-\langle H\rangle) / \sqrt{2 \operatorname{var}(H)}$,

$$
\frac{\mathcal{C}}{\mathcal{F}}=\frac{2}{\pi}\left[1-2 \tilde{b}^{2}(1-2 / \pi)+O\left(\ln ^{-1} N\right)+O\left(\tilde{b}^{3}\right)\right],
$$

so, as in Sec. IV A, for, e.g., $|b-\langle H\rangle|=0.3 \sqrt{\operatorname{var}(H)}$ (and $N \gg 1), \mathcal{C} / \mathcal{F}$ will degrade by only approximately $3.3 \%$.

\section{Critical systems}

The thermal Fisher information (3) is proportional to the heat capacity of the system, which scales as $C=N c(\beta)$, where $c(\beta)$ is the specific heat. For noncritical systems, $c(\beta)$ is intensive. However, at a finite-temperature phase transition, it diverges as the temperature of the system approaches the critical temperature $T_{c}>0$, according to $c(\beta) \propto|t|^{-\alpha}$, where $t:=\left(\beta-\beta_{c}\right) / \beta_{c}$ and $\alpha \geq 0$ is the so-called critical exponent [66]. When $\alpha=0$, the divergence is logarithmic: $c(\beta) \propto \ln |t|^{-1}$ [66]. In large but finite systems, there are of course no infinities, and at the phase transition point, $c_{N}\left(\beta_{c}\right) \propto N^{\alpha /(2-\alpha)}$ when $\alpha>0$ [67] and $c_{N}\left(\beta_{c}\right) \propto \ln N$ when $\alpha=0[67,68]$. Since $c_{N}=$ $\left(\beta^{2} \operatorname{var}(H)\right) / N$ [Eq. (3)], the divergence of $c_{N}\left(\beta_{c}\right)$ with $N$ implies that critical systems do not satisfy condition (ii) in Sec. IV B. In general, critical systems also feature diverging correlation lengths [66], thereby violating condition (i) in Sec. IV B as well. Therefore, the many-body Berry-Esseen theorem becomes inapplicable for critical systems.

In Appendix F, building on several rigorous results on translation-invariant lattices with finite-range interactions in Refs. [61,69,70], we develop an alternative approach toward determining the energy distribution of such lattices in arbitrary spatial dimensions. First of all, for noncritical lattices, we show that the energy distribution approximates a Gaussian in a way that complements the many-body Berry-Esseen theorem [59,60]. Moreover, for this wide but specific class of lattices, our approach allows us to access the energy distribution even at criticality.

For critical lattices with $\alpha=0$, we show in Appendix F 2 a that the energy distribution still tends to a Gaussian in the $N \rightarrow \infty$ limit; however, the convergence does not include the tails of the distribution, which are $O(\sqrt{N})$ standard deviations away from $\langle H\rangle$. In a sense, for translationinvariant lattices, this result suggests that the Gaussianity of the distribution holds beyond assumptions (i) and (ii) above $[59,60]$. Thus, Eq. (36) applies in the thermodynamic limit, both at criticality (with $\alpha=0$ ) and away from it. We illustrate these ideas in Sec. IV C1 with a detailed study on the classical 2D Ising model on a square lattice, a paradigmatic model with $\alpha=0$.

The case $1>\alpha>0$ is treated in Appendix F $2 \mathrm{~b}$. We show that the energy distribution is Gaussian only in a neighborhood of the peak that is much smaller than the standard deviation. Hence, it is not Gaussian as a whole. Notwithstanding, we show that it is unimodal with exponentially decaying tails, which means that the considerations in Appendix $\mathrm{C}$ are applicable; that is, a two-bin measurements with the boundary placed at $\langle H\rangle$ will yield $\mathcal{C}$ that scales proportionally to $\mathcal{F}$. In other words, since $\mathcal{F}=\beta^{2} N c_{N}$, we will have $\mathcal{C} \propto \beta^{2} N^{2 /(2-\alpha)}$.

\section{Two-dimensional Ising model}

The square-lattice two-dimensional (2D) Ising model is defined on an $L \times L$ square lattice where each site $i$ is characterized by a Pauli matrix $\sigma_{z}^{i}$, with $i=1, \ldots, N\left(N=L^{2}\right)$. The spins interact according to the Hamiltonian

$$
H=-J \sum_{\langle i, j\rangle} \sigma_{z}^{i} \sigma_{z}^{j},
$$

where the sum is over all nearest neighbors. Since the interactions involve only $\sigma_{z}$ operators, the Hamiltonian is already diagonal in the computational basis, with energy eigenvalues

$$
E(\boldsymbol{\sigma})=-J \sum_{\langle i, j\rangle} \sigma_{i} \sigma_{j}
$$

where $\sigma=\left(\sigma_{1}, \ldots, \sigma_{N}\right)$ and $\sigma_{i}= \pm 1$ are the eigenvalues of $\sigma_{z}^{i}$. Here we impose periodic boundary conditions. The model presents a phase transition at $T_{c} / J=2 / \ln (1+\sqrt{2})$ $[71,73,74]$. This can be seen, for instance, in terms of the magnetization $m=(1 / N) \sum_{i}\left\langle\sigma_{i}\right\rangle$, as plotted in Fig. 6(a).

For not very large $N$, the full energy distribution $q(E)$ can be computed exactly with use of a method developed in Ref. [72]. Results for $L=8,16,32$, and 64 are shown in Figs. 6(b)-6(e). Although the distribution is irregular for small sizes, it can be seen that the distribution visually appears to approach a Gaussian as the lattice size increases.

To rigorously prove that this is indeed the case, one needs to show that the higher-order $(\geq 3)$ cumulants of the energy distribution, $\kappa_{k}$, become irrelevant as $N$ becomes large. As discussed in Appendix F 3, the cumulants of the energy distribution can be found from the free energy $F_{N}$ through the simple relation

$$
\kappa_{k}=(-1)^{k-1} \frac{\partial^{k}\left(\beta F_{N}\right)}{\partial \beta^{k}} .
$$

Moreover, for the 2D Ising model, an exact expression for $F_{N}$, for finite $N$, is available [75]. Using these facts, we 

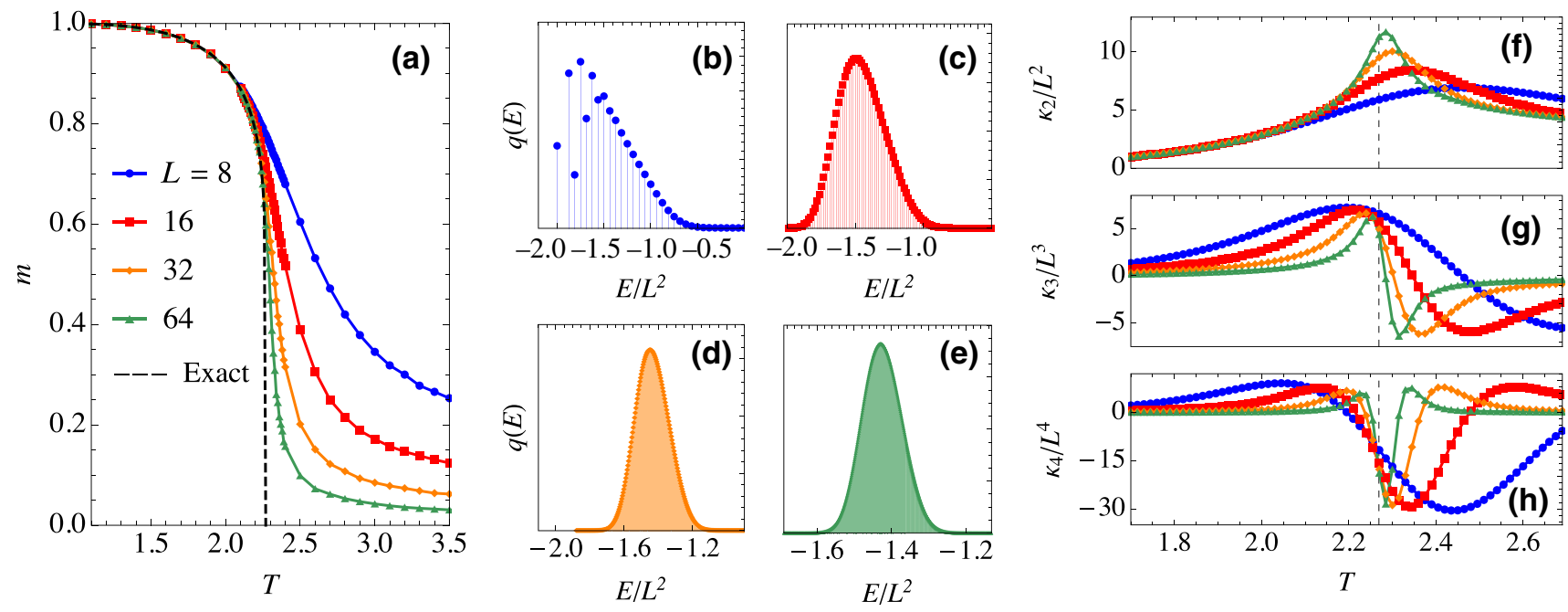

FIG. 6. Classical two-dimensional Ising model [Eq. (39)]. (a) Magnetization as a function of temperature for different lattice sizes, showing the phase transition at $T_{c} / J=2.26919$. The dashed curve corresponds to the thermodynamic limit (where the exact solution is $m=\left[1-\sinh ^{-4}(2 J / T)\right]^{1 / 8}$ [71]). (b)-(e) Exact energy distributions $q(E)$ for $T=T_{c}$ computed using the method in Ref. [72]. Each curve corresponds to a different lattice size, $L=8,16,32$, and 64 [same color code as in (a)]. (f)-(h) Cumulants $\kappa_{2}, \kappa_{3}$, and $\kappa_{4}$ of $q(E)$ as a function of temperature for different lattice sizes. The third and fourth cumulants scale, respectively, as $N^{3 / 2}$ and $N^{2}$, where $N=L^{2}$ is the number of sites. The second cumulant, on the other hand, scales as $N \ln N$.

show in Appendix F 3 that $\kappa_{2} \propto N \ln N[66,68]$, while $\kappa_{k} \propto$ $N^{k / 2}$ for $k \geq 3$ [these results are also illustrated in Figs. $6(\mathrm{f})-6(\mathrm{~h})]$. As a consequence, we therefore have

$$
\frac{\kappa_{k}^{1 / k}}{\kappa_{2}^{1 / 2}} \propto \ln ^{-1 / 2} N,
$$

showing that the higher-order cumulants do indeed become negligible as compared with $\kappa_{2}=\operatorname{var}(H)$; that is, the distribution tends to a Gaussian as $N \rightarrow \infty$.

The previous discussion refers to the scaling in the vicinity of the critical point. Away from it, because of the extensivity and analyticity of $F_{N}$ in the limit of $N \rightarrow \infty$, we simply have from Eq. (40) that $\kappa_{k} \propto N$ for $k \geq 1$. Hence, $\kappa_{k}^{1 / k} / \kappa_{2}^{1 / 2} \propto N^{-(k-2) /(2 k)}$; that is, away from criticality, $q(E)$ approaches its Gaussian limit polynomially, as compared with the slow logarithmic convergence at criticality.

\section{PROBE-BASED MEASUREMENTS}

In contrast to the previous section, where we allowed for arbitrary global measurements, in this section, we will look at measurement schemes (both idealized and more realistic) that can be realized by the interaction of a probe with the system, possibly some auxiliary system of arbitrary size, and the subsequent measurement of the probe alone. We compare the performance of such probe-based measurements with the upper bounds obtained in Sec. III B.

First of all, we observe that the maximal thermometric precision achievable by measuring a $d$-dimensional probe
$P$ that has unitarily interacted with the system $S$ in a thermal state $\tau$, and an auxiliary system $\mathcal{A}$ in some state $\rho_{\mathcal{A}}$, is the same as the maximal precision of a $d$-outcome measurement on $S$. Here we assume that $d<D$, because otherwise one can simply transfer all of the state of $S$ - and the (Fisher) information on $\beta, \mathcal{F}$, along with it - to $P$; however, when $d<D$, even the best possible strategy of encoding the state of $S$ into that of $P$ will result in losses. Indeed, if the initial state of $P$ is some $\sigma$, then, whatever the optimal unitary $U$, standard quantum metrology tells us [76] that the optimal POVM on

$$
\sigma^{\prime}=\operatorname{Tr}_{S \mathcal{A}}\left\{U\left(\sigma \otimes \tau \otimes \rho_{\mathcal{A}}\right) U^{\dagger}\right\}
$$

will have to have $d$ outcomes. On the other hand, the probability distribution generated by any $d$-outcome POVM on $\sigma^{\prime}$ can also be generated by a $d$-outcome POVM on $S$. Thus, denoting the quantum Fisher information of $\sigma^{\prime}$ on $\beta$ by $\mathcal{C}^{(P)}$, we have $\mathcal{C}^{(P)} \leq \mathcal{C}$.

To show that $\mathcal{C}^{(P)}=\mathcal{C}$, we note that $\mathcal{C}$ is delivered by a projective measurement on the system corresponding to some binning $I_{1} \cup \cdots \cup I_{d}$ that yields measurement statistics $p_{\alpha}=\sum_{q_{j} \in I_{\alpha}} q_{j}$. We choose $\sigma=|1\rangle\langle 1|$ so that, in the $\left\{|\alpha\rangle \otimes\left|E_{j}\right\rangle\right\}$ basis, $\sigma \otimes \tau=\operatorname{diag}(\overrightarrow{\mathbf{q}}, \overrightarrow{\mathbf{0}}, \ldots, \overrightarrow{\mathbf{0}})$. Here $\overrightarrow{\mathbf{q}}=\left(q_{1}, \ldots, q_{D}\right)$ and $\overrightarrow{\mathbf{0}}$ is made of $D$ zeroes. Then the permutation unitary acting on $\sigma \otimes \tau$ that permutes all the $q_{j}$ 's in $I_{2}$ from $\overrightarrow{\mathbf{q}}$ with some of the zeroes from the $\overrightarrow{\mathbf{0}}$ next to $\overrightarrow{\mathbf{q}}$, all the $q_{j}$ 's in $I_{3}$ with zeroes from the second $\overrightarrow{\mathbf{0}}$, etc., will ren$\operatorname{der} \sigma^{\prime}=\operatorname{diag}\left(p_{1}, \ldots, p_{d}\right)$. This distribution will produce a $\mathcal{C}^{(P)}$ that is equal to $\mathcal{C}$. Note that, to show that $\mathcal{C}^{(P)}$ can be 
made equal to $\mathcal{C}$, there was no need to involve any auxiliary systems.

For the case of $d=2$, where the optimal POVM on $S$ is defined by the bins $I_{1}=\left\{E_{j}: E_{j}<b\right\}$ and $I_{2}=\left\{E_{j}\right.$ : $\left.E_{j} \geq b\right\}$, with $b$ being the boundary, we now show that such a permutation can be generated by the quantum-opticsinspired Hamiltonian

$$
\begin{aligned}
H= & \sum_{k=1}^{D} E_{k}\left|E_{k}\right\rangle\left\langle E_{k}|+b| \Uparrow\right\rangle\langle\Uparrow| \\
& +\lambda \sum_{E_{k} \leq E_{D}-b}\left(|\Downarrow\rangle\left\langle\Uparrow|\otimes| E_{k}+b\right\rangle\left\langle E_{k}\right|+\text { H.c. }\right),
\end{aligned}
$$

where $|\Downarrow\rangle$ and $|\Uparrow\rangle$ are the eigenstates of the probe spin, with the corresponding eigenvalues $E_{\Downarrow}=0$ and $E_{\Uparrow}=b$. This Hamiltonian may not be easily realizable in practice. However, the point is that, as we show, it is guaranteed to provide the best possible precision using a two-level probe. This can then be used as a benchmark to compare against when other interactions are used. Furthermore, we take the system's ground state to be at energy $E_{1}=0$, and since ultimately we are going to be interested in the case where the system's energy spectrum is effectively continuous, we also assume that $\left|E_{k}+b\right\rangle$ is a valid eigenstate.

We initialize the system and the probe in the joint state $\rho(0)=|\Downarrow\rangle\langle\Downarrow| \otimes \tau$. The simplest way to characterize its evolution under $H$ is to describe how the pure states constituting it, $\left|\Psi_{j}(0)\right\rangle=|\Downarrow\rangle \otimes\left|E_{j}\right\rangle$, evolve under $H$. It is easy to show that, in the interaction picture (labeled by the superscript I), $\left|\Psi_{j}^{\mathrm{I}}(t)\right\rangle=|\Downarrow\rangle \otimes\left|E_{j}\right\rangle$ for $E_{j}<b$, and

$$
\left|\Psi_{j}^{\mathrm{I}}(t)\right\rangle=\cos (\lambda t)|\Downarrow\rangle \otimes\left|E_{j}\right\rangle-i \sin (\lambda t)|\Uparrow\rangle \otimes\left|E_{j}-b\right\rangle
$$

for $E_{j} \geq b$. Thus, transitioning back to the Schrödinger picture, from $\rho(t)=\sum_{j} \frac{1}{Z} e^{-\beta E_{j}}\left|\Psi_{j}(t)\right\rangle\left\langle\Psi_{j}(t)\right|$, we find the probability of finding the probe qubit in the spin-up state, $\mathbb{P}_{\Uparrow}$, when measuring it at time $t$ to be

$$
\mathbb{P}_{\Uparrow}(t)=\sin ^{2}(\lambda t) \sum_{E_{j} \geq b} \frac{e^{-\beta E_{j}}}{Z} .
$$

Hence, for $t_{\text {meas }}=\pi /(2 \lambda)$, the ideal projective measurement of the probe qubit's spin produces a probability distribution identical to that produced by the ideal binary measurement of the system corresponding to the binning $I_{1} \cup I_{2}$.

Note that realizing this idealized scheme experimentally is far from being straightforward. Therefore, in the next subsection, we study a specific model realization, and describe both its capabilities and its limitations.

\section{A. Jaynes-Cummings model}

As a specific illustration of quantum probe-based thermometry, we consider an experimentally relevant system consisting of a superfluid Bose-Einstein condensate reservoir in a shallow confining trap interacting with an atomic quantum dot [45]. Generally, the physics of this system is well captured by a spin-boson model, in which the atomic quantum dot interacts with the phononic excitations of the Bose-Einstein condensate superfluid. Given suitably engineered boundary conditions, the spectral density will be such that a quantum dot with frequency $\omega_{d}$ will in effect couple only to the phonon modes that come closest to being resonant with the quantum dot frequency (for simplicity we suppose all relevant phonon modes have the same frequency $\omega_{a}$ ).

We can then ask the question of how well one can estimate the Bose-Einstein condensate temperature by measurements on the quantum dot probe. In Fig. 7(a) we plot the ratio of coarse-grained Fisher information and the thermal Fisher information for the optimal binary measurement within the effectively resonant subspace. The ratio is given as a function of the number of effectively resonant modes, which would be expected to increase in proportion to the width of the spectral density. From Fig. 7(a) we see that the ratio approaches a value of 0.64 as the number of modes increases. This provides an optimal value against which to compare specific binary measurement strategies.
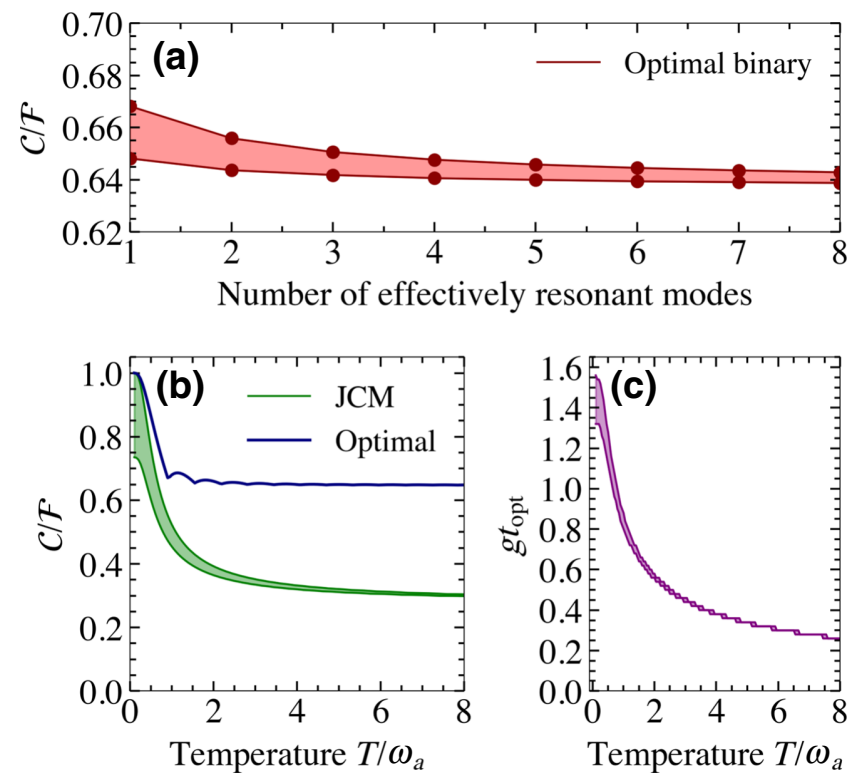

FIG. 7. (a) Optimal binary-outcome thermometry on a collection of bosonic modes of frequency $\omega_{a}$ as a function of the number of oscillators. The top line corresponds to $\beta \omega_{a}=0.7$, the bottom line corresponds to $\beta \omega_{a}=0.1$, and intermediate temperatures are contained within the shaded area. (b) Comparison between the optimal binary measurement strategy and the two-level Jaynes-Cummings model probe, optimized over the measurement time $g t$, in the single oscillator case. The shaded area represents the range $\delta / g=[0,1.2]$ of the detuning. (c) Optimal measurement time $\left(g t_{\text {opt }}\right)$ as a function of temperature for the Jaynes-Cummings model. 
We see that the obtained ratio agrees with the optimal ratio found for a binary measurement on a system described by a Gaussian density of states.

If we now consider the specific case where the effectively resonant subspace consists of a single phononic mode, and furthermore make a rotating wave approximation, the resulting system is modeled by the paradigmatic Jaynes-Cummings Hamiltonian [77]:

$$
H=\omega_{d} \sigma^{\dagger} \sigma+\omega_{a} a^{\dagger} a+g\left(\sigma^{\dagger} a+\sigma a^{\dagger}\right),
$$

where $a$ and $a^{\dagger}$ are the creation and annihilation operators of the bosonic cavity mode, $g$ is the coupling strength, and $\sigma=|g\rangle\langle e|$, where the excited and ground states of the quantum dot are denoted by $|e\rangle$ and $|g\rangle$, respectively. Experimental work has shown how such models arise for specific thermometry protocols [6], and our results makes it possible to evaluate how close such a strategy is to being optimal.

Consider a measurement protocol in which the quantum dot is initialized in its ground state. The quantum dot then evolves jointly with the Bose-Einstein condensate for a time $t$, after which the probability of finding the quantum dot in its excited state is given by

$$
\mathbb{P}_{e}(t)=\sum_{n=0}^{\infty} \frac{e^{-\beta \omega_{a}(n+1)}}{Z_{\beta}} \frac{g^{2}(n+1)}{\lambda_{n}^{2}} \sin ^{2}\left(\lambda_{n} t\right),
$$

where we have defined $\lambda_{n}=\sqrt{\delta^{2} / 4+g^{2}(n+1)}$ in terms of the detuning $\delta=\omega_{d}-\omega_{a}$. From this probability we can compute the coarse-grained Fisher information; see also Ref. [22]. In Fig. 7(b) we show the ratio of the coarsegrained Fisher information computed from Eq. (46) and optimized over the measurement time at each temperature and the thermal Fisher information of the phononic mode itself. Inspection of the results shows that the probe-based measurement gives a Fisher information that never falls below $45 \%$ of the optimal binary measurement strategy. In Fig. 7(c) we plot the optimal measurement time as a function of temperature, and we observe an inverse relationship between the optimal measurement time and the temperature. Notice that as the temperature approaches absolute zero, the optimal measurement time for zero detuning approaches $\pi /(2 g)$, in agreement with previous results [22].

It is interesting to note that similar considerations for the Fisher information were obtained for temperature measurements of micromechanical resonators via a qubit probe, whose interaction can also be described by the Jaynes-Cummings Hamiltonian (45) [22]; and more general interaction Hamiltonians, either dropping the rotating wave approximation or considering interactions far off resonance, have also been considered [13,23]. In all such cases, our considerations provide upper bounds on the maximal precision estimation with a qubit probe, as shown in Fig. 7. Indeed, the strength of our bounds is that they apply to arbitrary nonequilibrium strategies.

\section{CONCLUSIONS}

We have considered the precision limits on temperature estimation when having access to coarse-grained measurements that have at most $d$ outcomes. Using tools from signal processing, we derived the structure of the optimal POVM measurement. We applied these abstract considerations to two physically relevant scenarios: temperature measurements of many-body systems and nonequilibrium thermometry.

For many-body systems, we considered spin lattices, both away from and at criticality, and found that the Fisher information $\mathcal{C}$ can grow extensively with the system size even when $d$ does not. In particular, for $d=2$, we found that it is in principle possible that $\mathcal{C} / \mathcal{F} \approx 2 / \pi$ in the thermodynamic limit $N \rightarrow \infty$ even for systems at criticality. While this will decrease for realistic strategies where the POVM are smoothed out (see the discussion in Sec. III D and Fig. 4 specifically), we expect that the extensive scaling will be preserved as long as the binary measurement can distinguish system energies that are $O(\sqrt{\operatorname{var}(H)})$ apart (see the discussion on displaced boundary in Secs. IV A and IV B).

Along the way, we also derived new results for the energy distribution of many-body systems in the regime of criticality, which might be of independent interest. These generic considerations were illustrated on the 2D Ising model, the energy distribution of which becomes well approximated by a Gaussian distribution except in the tails of the distribution. We expect more pronounced non-Gaussian features in the energy distribution of other critical models, which we leave as interesting future research.

For nonequilibrium thermometry, we used our results to devise the optimal probe-system interaction and interrogation time, thus providing general guidelines for the design of optimal nonequilibrium thermometry strategies. This result also provides an upper bound on specific experimentally motivated protocols. We illustrated this for a temperature measurement of a Bose-Einstein condensate through a quantum dot via a Jaynes-Cummings interaction $[22,23]$. It remains an exciting open question to find a realistic implementation of the optimal probe-sample interaction (43).

Lastly, in this work, we focused on asymptotic metrology, where one has access to full measurement statistics and can possibly run the experiment many times. This may not always be feasible in practice, and the Fisher information may then no longer be an adequate precision quantifier. In such cases, alternative approaches are 
needed, such as global Bayesian estimation [78]. Analyzing the effect of coarse-graining in such situations is another interesting research direction.

\section{ACKNOWLEDGMENTS}

We sincerely thank Raffaele Salvia for pointing us to Ref. [56], from which it follows that consecutive binnings are optimal, as previously conjectured. We also gratefully acknowledge fruitful discussions with Raam Uzdin. M.R.J. and J.B.B. are supported by the Independent Research Fund Denmark. G.T.L. acknowledges the financial support of the São Paulo Funding Agency FAPESP (Grants No. 2017/50304-7, No. 2017/07973-5, and No. 2018/128130 ) and the Brazilian funding agency $\mathrm{CNPq}$ (Grant No. INCT-IQ 246569/2014-0). M. P.-L. acknowledges funding from the Swiss National Science Foundation (Ambizione PZ00P2-186067). The authors acknowledge the International Centre for Theoretical Physics, in Trieste, where part of this work was developed, for both financial support and hospitality.

\section{APPENDIX A: OPTIMALITY OF CONSECUTIVE BINNING}

In this appendix, we apply Theorem 1 in Ref. [56] to prove that the sets $I_{\alpha}$ maximizing the coarse-grained Fisher information $\mathcal{C}$ can be chosen to be consecutive. First, we state the theorem mentioned and then we show how it applies to our problem.

Theorem 1 (Theorem 1 in Ref. [56]). Let $a_{1}, \ldots, a_{D}$ and $b_{1}, \ldots, b_{D}$ be real numbers such that either $a_{i} \geq 0 \forall i$ or $b_{i} \geq 0 \forall i$ and

$$
\frac{a_{1}}{b_{1}} \leq \cdots \leq \frac{a_{D}}{b_{D}} .
$$

If some $b_{i}=0$, then $a_{i} / b_{i}$ is defined as $\operatorname{sgn} a_{i} \times \infty$ when $a_{i} \neq 0$. When $a_{i}$ is also $0, a_{i} / b_{i}$ is defined arbitrarily to satisfy Eq. (A1). Next, for a partitioning of the set $\{i\}_{i=1}^{D}$ into $d$ nonoverlapping subsets $\left\{I_{\alpha}\right\}_{\alpha=1}^{d}, \bigcup_{\alpha=1}^{d} I_{\alpha}=\{i\}_{i=1}^{D}$, define $a_{I_{\alpha}}:=\sum_{i \in I_{\alpha}} a_{i}$ and $b_{I_{\alpha}}:=\sum_{i \in I_{\alpha}} b_{i}$. Then the maximum of an objective function

$$
h\left(a_{I_{1}}, b_{I_{1}}, \ldots, a_{I_{d}}, b_{I_{d}}\right)
$$

over all possible partitionings $\left\{I_{\alpha}\right\}_{\alpha=1}^{d}$ is delivered by a partitioning into consecutive subsets if $h$ is a jointly convex function of all of its $2 d$ arguments. (A subset of $\{i\}_{i=1}^{D}$ is called "consecutive" if its elements are consecutive numbers.)

Returning to our problem, we assign $a_{i}=q_{i}\left(E_{i}-\langle H\rangle\right)$ and $b_{i}=q_{i}$. Now we immediately see that $b_{i}>0$ for all $i$ 's and $a_{i} / b_{i}=E_{i}-\langle H\rangle$ satisfies the condition (A1) in view of Eq. (14). In terms of $a_{I_{\alpha}}$ and $b_{I_{\alpha}}$, our objective function, $\mathcal{C}$ (corresponding to $h$ in the theorem), as defined by Eq. (17), reads

$$
\mathcal{C}\left(a_{I_{1}}, b_{I_{1}}, \ldots, a_{I_{d}}, b_{I_{d}}\right)=\sum_{\alpha=1}^{d} \frac{a_{I_{\alpha}}^{2}}{b_{I_{\alpha}}}
$$

In view of the joint convexity of the function $a^{2} / b$ [55], we immediately see that $\mathcal{C}\left(a_{I_{1}}, b_{I_{1}}, \ldots, a_{I_{d}}, b_{I_{d}}\right)$ is a jointly convex function of all of its $2 d$ arguments.

So, having shown that all the conditions of Theorem 1 are met for our problem, we have thus proven that the maximum of $\mathcal{C}$ over all partitions (equivalent to nonoverlapping bins) $\left\{I_{\alpha}\right\}_{\alpha=1}^{d}$ is delivered by a consecutive partitioning.

Lastly, we note that the optimality of consecutive partitioning does not preclude the possibility that some other, nonconsecutive partitioning delivers the same maximum. Direct numerical checks show that for our particular case such coincidences do indeed happen.

\section{APPENDIX B: NONINTERACTING QUBITS IN THE LARGE- $N$ LIMIT}

In the limit of a large number of qubits, we can approximate the sums in Eq. (26) by integrals (using the de Moivre-Laplace theorem) and obtain the approximate probabilities and bin energies

$$
\begin{aligned}
p_{\alpha}= & \frac{1}{2}\left[\operatorname{erf}\left(\frac{N s-b_{\alpha-1}}{\sqrt{2 N r s}}\right)-\operatorname{erf}\left(\frac{N s-b_{\alpha}}{\sqrt{2 N r s}}\right)\right], \\
\epsilon_{\alpha}= & \langle H\rangle-\frac{\sqrt{N r s}}{\sqrt{2 \pi} p_{\alpha}}\left[\exp \left(-\frac{\left(b_{\alpha}-N s\right)^{2}}{2 N r s}\right)\right. \\
& \left.-\exp \left(-\frac{\left(b_{\alpha-1}-N s\right)^{2}}{2 N r s}\right)\right] .
\end{aligned}
$$

The case of binary measurements, $d=2$, is defined by a single bin boundary $b=b_{1}$. Assuming the system to be at a temperature such that $N s / r \gg 1$, substituting $p_{\alpha}$ and $\epsilon_{\alpha}$ into Eq. (17), we find

$$
\frac{\mathcal{C}}{\mathcal{F}} \approx \frac{2}{\pi} \frac{\exp \left(-\frac{(N s-b)^{2}}{N r s}\right)}{1-\operatorname{erf}\left(\frac{N s-b}{\sqrt{2 N r s}}\right)^{2}} .
$$

The ratio takes its maximal value for $b=N s$, which is also a solution to Eq. (24). In this case we therefore arrive at Eq. (27). This is consistent with Eq. (36) in view of the de Moivre-Laplace theorem, which almost straightforwardly states that the energy distribution of $N \gg 1$ thermally identical noninteracting qubits is a discrete Gaussian. Thus, we find that in the large- $N$ limit for 
a binary measurement, the optimal partition is at the average energy, and a very appealing feature is that such a measurement provides Fisher information that is already $2 / \pi \approx 64 \%$ of the maximal possible value, independently of the system size $N$.

\section{APPENDIX C: PROPORTIONALITY $\mathcal{C} \propto \mathcal{F}$ FOR TWO-BIN MEASUREMENTS ON SHORT TAIL DISTRIBUTIONS}

In this appendix we show that a proportionality of the form $\mathcal{C} \propto \mathcal{F}$ is generically true for two-bin measurement, in which the separation of the bins is at the average energy $\langle H\rangle$, for any distribution that decays sufficiently quickly [see Eq. (C4)]. By "energy distribution" we understand $q(E)=\Omega(E)\left(e^{-\beta E} / Z\right)$ [Eq. (20)], and not just $e^{-\beta E}$. Indeed, since $\beta>0, e^{-\beta E}$ must decay monotonically with energy. But $\Omega(E)$ may concentrate energy in different sectors, which can cause the overall distribution $q(E)$ to have arbitrary shapes. Indeed, as discussed in Sec. IV, quite often $q(E)$ will have a Gaussian shape or, more generally, a short tail distribution.

For a dichotomic measurement, with bin position at $b=\langle H\rangle$, the coarse-grained Fisher information (17) can be written, with some rearrangements, as

$$
\mathcal{C}=\beta^{4} \frac{\mathfrak{E}^{2}}{p_{+}\left(1-p_{+}\right)}
$$

where

$$
\begin{gathered}
p_{+}=\int_{\langle H\rangle}^{\infty} d E q(E) \\
\mathfrak{E}=\int_{\langle H\rangle}^{\infty} d E(E-\langle H\rangle) q(E)=\int_{0}^{\infty} d E E \hat{q}(E) .
\end{gathered}
$$

Here, to simplify notation, we also introduced $\hat{q}(E)=$ $q(E+\langle H\rangle)$.

Now we require - and this is the only requirement we impose - that there exists a fixed (i.e., independent of $N$ ) number $\lambda>1$ such that

$$
\int_{I_{3}} d E E^{2} \hat{q}(E) \leq A \operatorname{var}(H),
$$

where

$$
0 \leq A<\frac{1}{2}
$$

and

$$
I_{3}=\{E:|E|>\lambda \sqrt{\operatorname{var}(H)}\} .
$$

These conditions are generically expected to be satisfied by unimodal distributions with fast decaying tails; however, unimodality is not a requirement as long as the above requirement is met.

We further pick

$$
0<\lambda_{0}<\frac{1}{2}
$$

and define

$$
I_{1}=\left\{E:|E| \leq \lambda_{0} \sqrt{\operatorname{var}(H)}\right\} .
$$

We designate the remaining interval as

$$
I_{2}=\left\{E: \lambda_{0} \sqrt{\operatorname{var}(H)}<|E| \leq \lambda \sqrt{\operatorname{var}(H)}\right\},
$$

and we further define

$$
p_{k}=\int_{I_{k}} d E \hat{q}(E), \quad k=1,2,3
$$

Now, by Eq. (C8),

$$
\int_{I_{1}} d E E^{2} \hat{q}(E) \leq \lambda_{0}^{2} \operatorname{var}(H) p_{1}=\lambda_{0}^{2} \operatorname{var}(H)\left(1-p_{2}-p_{3}\right) .
$$

Then, again, by the definition of Eq. (C9),

$\lambda^{2} \operatorname{var}(H) p_{2} \geq \int_{I_{2}} d E E^{2} \hat{q}(E)=\operatorname{var}(H)-\int_{I_{1} \cup I_{3}} d E E^{2} \hat{q}(E)$.

Invoking Eqs. (C4) and (C11), we rewrite Eq. (C12) as

$$
\lambda^{2} \operatorname{var}(H) p_{2} \geq\left(1-A-\lambda_{0}^{2}\right) \operatorname{var}(H)+\lambda_{0}^{2} \operatorname{var}(H) p_{2},
$$

which gives

$$
p_{2} \geq \frac{1-A-\lambda_{0}^{2}}{\lambda^{2}-\lambda_{0}^{2}}
$$

With this, we immediately find that

$$
\mathfrak{E}=\int_{0}^{\infty} d E E \hat{q}(E) \geq \int_{I_{2}} d E E \hat{q}(E) \geq \lambda_{0} \sqrt{\operatorname{var}(H)} p_{2},
$$

and thus

$$
\mathfrak{E} \geq \lambda_{0} \frac{1-A-\lambda_{0}^{2}}{\lambda^{2}-\lambda_{0}^{2}} \sqrt{\operatorname{var}(H)}
$$


Since $p_{+}\left(1-p_{+}\right) \leq 1 / 4$, from Eq. (C1), we have

$$
\mathcal{C} \geq \Xi \beta^{4} \operatorname{var}(H)
$$

where

$$
\Xi=4 \lambda_{0}^{2}\left(\frac{1-A-\lambda_{0}^{2}}{\lambda^{2}-\lambda_{0}^{2}}\right)^{2}, \quad 0<\Xi<1 .
$$

Keeping in mind Eq. (3), we thus have

$$
\Xi \mathcal{F} \leq \mathcal{C} \leq \mathcal{F} .
$$

The bound above, the purpose of which is solely to prove the scaling of $\mathcal{C}$, may be rather loose, and it is of course desirable to find tighter bounds. We leave this task for future work.

\section{APPENDIX D: EXAMPLE OF SMALL FISHER INFORMATION}

In this appendix we construct an example for which the scaling $\mathcal{C} \propto \mathcal{F}$, found in several examples throughout the main text, breaks down. Albeit rather artificial, the goal of this construction is to illustrate that, although occurring generically, the relation $\mathcal{C} \propto \mathcal{F}$ is not universal.

To construct our example, we take $N$ two-level systems, the total Hamiltonian of which is such that the density of states has four sharp peaks, approaching

$$
\begin{aligned}
\Omega_{N}(E)= & \vartheta_{N, \epsilon} \delta(E-\epsilon)+\vartheta_{N,-\epsilon} \delta(E+\epsilon) \\
& +\vartheta_{N, t N} \delta(E-t N)+\vartheta_{N,-t N} \delta(E+t N)
\end{aligned}
$$

as $N \rightarrow \infty$, where $t>0$ and $\epsilon>0$ are some fixed values of energy and the quantities $\vartheta_{N, \pm \epsilon}$ and $\vartheta_{N, \pm t N}$ are also positive. By definition, $\int d E \Omega_{N}(E)=2^{N}$, so

$$
\vartheta_{N, \epsilon}+\vartheta_{N,-\epsilon}+\vartheta_{N, t N}+\vartheta_{N,-t N}=2^{N} .
$$

We emphasize that the distributions are discrete and become continuous in the $N \rightarrow \infty$ limit, and the $\delta$ functions above are also to be understood as sharp peaks that approach the $\delta$ function in the $N \rightarrow \infty$ limit.

Finally, we require that the effectively discrete energy distribution $q_{N}(E)=(1 / Z) e^{-\beta E} \Omega_{N}(E)$ [cf. Eq. (20)] is

$$
q_{N, E}= \begin{cases}\frac{1}{2}-\frac{1}{N}, & E= \pm \epsilon, \\ \frac{1}{N}, & E= \pm t N,\end{cases}
$$

which corresponds to the following choice of the quantities $\vartheta_{N, E}$ :

$$
\vartheta_{N, \pm \epsilon}=\left(\frac{1}{2}-\frac{1}{N}\right) e^{ \pm \beta \epsilon} Z
$$

$$
\vartheta_{N, \pm t N}=\frac{1}{N} e^{ \pm \beta t N} Z
$$

where, according to Eq. (D2), $Z$ should be

$$
Z=\frac{2^{N}}{(2 / N) \cosh (\beta t N)+(1-2 / N) \cosh (\beta \epsilon)} .
$$

Now, it is straightforward to see that the average energy corresponding to $q_{N, E}$ is zero. Thus, the thermal Fisher information will be

$$
\mathcal{F}=\beta^{4} \sum_{E= \pm \epsilon, \pm t N} q_{N, E} E^{2}=\beta^{4}\left[2 t^{2} N+\left(1-\frac{2}{N}\right) \epsilon^{2}\right] .
$$

Furthermore, considering two-outcome measurements with the optimal boundary being at the average energy (in this case 0 ), the bin probabilities will be $p_{1}=p_{2}=1 / 2$ [cf. Eq. (15)] and the bin energies [cf. Eq. (16)] will be

$$
\epsilon_{1}=-\epsilon_{2}=2\left[t+\epsilon\left(\frac{1}{2}-\frac{1}{N}\right)\right]
$$

hence, according to Eq. (17),

$$
\mathcal{C}=4 \beta^{4}\left[t+\epsilon\left(\frac{1}{2}-\frac{1}{N}\right)\right]^{2} .
$$

Thus,

$$
\frac{\mathcal{C}}{\mathcal{F}}=\frac{4\left[t+\epsilon\left(\frac{1}{2}-\frac{1}{N}\right)\right]^{2}}{2 t^{2} N+\left(1-\frac{2}{N}\right) \epsilon^{2}},
$$

which for fixed $\epsilon$ and $t$ scales proportionally to $1 / N$, which strongly breaks the $\mathcal{C} \propto \mathcal{F}$ relation.

\section{APPENDIX E: PROOF OF Eq. (36)}

To prove Eq. (36), we first state the quantum BerryEsseen theorem proven in Refs. [59,60] for lattices with finite-range interactions. The theorem requires the following two assumptions about the state of the system $\rho$ :

(i) The state has exponentially decaying correlations: for arbitrary regions $\mathcal{X}, \mathcal{Y}$ separated by a distance $l$ in the lattice and some constant $\xi$

$$
\max _{X \in \mathcal{X}, Y \in \mathcal{Y}}\left|\frac{\langle X \otimes Y\rangle-\langle X\rangle\langle Y\rangle}{\|X\|\|Y\|}\right| \leq e^{-l / \xi} .
$$

(ii) The variance in energy scales with the number of sites as $\operatorname{var}(H)=\left\langle H^{2}\right\rangle-\langle H\rangle^{2}=s^{2} N$. 
The system is an $N$-vertex lattice $\mathcal{L}_{N}$ (the vertices of which correspond to "particles") and is described by a locally bounded, finite-range interacting Hamiltonian:

$$
H=\sum_{v \in \mathcal{L}_{N}} H_{v}
$$

Each $H_{v}$ acts only on vertices the Manhattan distance of which from $v$ is $\leq z$, where $z$ is some fixed natural number that sets the (finite) range of the interactions within the lattice. Lastly, the theorem requires the Hamiltonian to be locally bounded: there exists a constant $\mathfrak{h}>0$ such that

$$
\left\|H_{v}\right\| \leq \mathfrak{h} \quad \forall v \in \mathcal{L}_{N}
$$

where $\|\cdot\|$ can be chosen to be, for example, the spectral norm ( $\mathfrak{h}$ will of course depend on the norm we choose).

Theorem 2 (Lemma 8 of Ref. [59]). Let $\rho$ be a state such that assumptions (i) and (ii) hold, and with a local Hamiltonian with uniformly bounded local terms, of a system of $N$ particles on a d-dimensional lattice. Given the cumulative function

$$
J(x)=\sum_{E_{i} \leq x}\left\langle E_{i}|\rho| E_{i}\right\rangle
$$

and the Gaussian cumulative function

$$
G(x)=\int_{-\infty}^{x} \frac{d t}{\sqrt{2 \pi \operatorname{var}(H)}} \exp \left(-\frac{(t-\langle H\rangle)^{2}}{2 \operatorname{var}(H)}\right),
$$

then

$$
\sup _{x}|J(x)-G(x)| \leq C_{0} \frac{\ln ^{2 \mathfrak{d}} N}{s^{3} \sqrt{N}}
$$

where $C_{0}$ is a constant.

Crucially, the constant $C_{0}$ does not depend on the system size but depends only on parameters such as the range of the Hamiltonian $(z)$, the lattice structure, or the correlation length $\xi$.

We can always set the energy of the ground state of $H$ to be zero. Moreover, keeping in mind Eqs. (E2) and (E3), we have $\|H\| \leq \sum_{v \in \mathcal{L}_{N}}\left\|H_{v}\right\| \leq \mathfrak{h} N$, meaning that the largest $E_{i}$ is $\leq \mathfrak{h} N$. Thus, the real range of energies in Eq. (E4) can be summarized as $E_{\min }:=\min \left\{E_{i}\right\}=0$ and $E_{\max }:=\max \left\{E_{i}\right\} \leq \mathfrak{h} N$, and for any $E \notin\left[E_{\min }, E_{\max }\right]$ one sets $q(E)=0$ so that integrals with infinite energy ranges are meaningful.

We now bound $\mathcal{C}$, assuming two partitions $I_{1}=(-\infty, b]$ and $I_{2}=(b, \infty)$ (i.e., $d=2$ ). We first estimate the probabilities $p_{1}$ and $p_{2}=1-p_{1}$, defined in Eq. (15), using
Theorem 2:

$$
p_{1}=\sum_{E_{i} \leq b} q_{i}=J(b)=G(b)+\varepsilon_{N},
$$

where

$$
\varepsilon_{N}=O\left(\frac{\ln ^{2 \mathfrak{d}} N}{\sqrt{N}}\right) .
$$

Next, we note that, since $\langle H\rangle=\sum_{E_{i}<b} q_{i} E_{i}+\sum_{E_{i} \geq b} q_{i} E_{i}$,

$$
\sum_{E_{i}<b} q_{i}\left(E_{i}-\langle H\rangle\right)=-\sum_{E_{i} \geq b} q_{i}\left(E_{i}-\langle H\rangle\right) .
$$

So, on our introducing

$$
\mathfrak{E}=\sum_{E_{i}-b \geq 0} q_{i}\left(E_{i}-\langle H\rangle\right)
$$

the bin energies, as given by Eq. (16), read

$$
\epsilon_{1}-\langle H\rangle=-\frac{\mathfrak{E}}{p_{1}} \quad \text { and } \quad \epsilon_{2}-\langle H\rangle=\frac{\mathfrak{E}}{p_{2}} .
$$

Therefore, according to Eq. (17), for the coarse-grained Fisher information, we obtain

$\mathcal{C}=\beta^{4}\left[p_{1}\left(\epsilon_{1}-\langle H\rangle\right)^{2}+p_{2}\left(\epsilon_{2}-\langle H\rangle\right)^{2}\right]=\beta^{4} \frac{\mathfrak{E}^{2}}{p_{1}\left(1-p_{1}\right)}$

Now, taking into account Eq. (E7), we can write

$$
\begin{aligned}
\frac{1}{p_{1}\left(1-p_{1}\right)}= & \frac{1}{G(b)[1-G(b)]} \\
& \times\left\{1+O\left(\varepsilon_{N}[2 G(b)-1]\right)+O\left(\varepsilon_{N}^{2}\right)\right\} .
\end{aligned}
$$

Thus, introducing

$$
\tilde{b}=\frac{b-\langle H\rangle}{\sqrt{2 \operatorname{var}(H)}}
$$

and noticing that

$$
G(b)=\frac{1}{2}+\frac{1}{2} \operatorname{erf} \tilde{b},
$$

similarly to Eq. (E12), we can write

$$
\mathcal{C}=\beta^{4} \frac{4 \mathfrak{E}^{2}}{1-(\operatorname{erf} \tilde{b})^{2}}\left[1+O\left(\epsilon_{N} \operatorname{erf} \tilde{b}\right)+O\left(\varepsilon_{N}^{2}\right)\right] .
$$


Turning to $\mathfrak{E}$, we rewrite Eq. (E10) as

$\mathfrak{E}=\sum_{E_{i}-b \geq 0} q_{i}\left(E_{i}-\langle H\rangle\right)=\sum_{l=0}^{\infty} \sum_{\Lambda l \leq E_{i}-b<\Lambda(l+1)} q_{i}\left(E_{i}-\langle H\rangle\right)$,

where $\Lambda>0$ is an arbitrary constant. With this, by choosing an arbitrary natural number $R$, we can lower-bound $\mathfrak{E}$ as

$$
\begin{aligned}
\mathfrak{E} & =\sum_{l=0}^{\infty} \sum_{\Lambda l \leq E_{i}-b<\Lambda(l+1)} q_{i}\left(E_{i}-\langle H\rangle\right) \\
& \geq \sum_{l=0}^{R} \sum_{\Lambda l \leq E_{i}-b<\Lambda(l+1)} q_{i}\left(E_{i}-\langle H\rangle\right) \\
& \geq \sum_{l=0}^{R}(\Lambda l+b-\langle H\rangle) \sum_{\Lambda l \leq E_{i}-b<\Lambda(l+1)} q_{i},
\end{aligned}
$$

which, on our introducing

$$
\widetilde{\Lambda}=\frac{\Lambda}{\sqrt{2 \operatorname{var}(H)}}
$$

reads

$$
\mathfrak{E} \geq \sqrt{2 \operatorname{var}(H)} \sum_{l=0}^{R}(\tilde{\Lambda} l+\tilde{b}) \sum_{\Lambda l \leq E_{i}-b \leq \Lambda(l+1)} q_{i} .
$$

Next, using Theorem 2, we arrive at the following estimate:

$$
\begin{aligned}
\sum_{\Lambda l \leq E_{i}-b<\Lambda(l+1)} q_{i} & =J[b+\Lambda(l+1)]-J(b+\Lambda l) \\
& =G[b+\Lambda(l+1)]-G(b+\Lambda l)+\varepsilon_{N} \\
& =\int_{b-\langle H\rangle+\Lambda l}^{b-\langle H\rangle+\Lambda(l+1)} \frac{d t}{\sqrt{2 \pi \operatorname{var}(H)}} e^{-\frac{t^{2}}{2 \operatorname{var}(H)}} \\
& +\varepsilon_{N}=\frac{1}{\sqrt{\pi}} \int_{\tilde{b}+\tilde{\Lambda} l}^{\tilde{b}+\tilde{\Lambda}(l+1)} d t e^{-t^{2}}+\varepsilon_{N} .
\end{aligned}
$$

Substituting Eq. (E20) into Eq. (E19), we obtain

$$
\begin{aligned}
\mathfrak{E} & \geq \sqrt{\frac{2 \operatorname{var}(H)}{\pi}} \sum_{l=0}^{R}(\tilde{b}+\widetilde{\Lambda} l) \int_{\tilde{b}+\tilde{\Lambda} l}^{\tilde{b}+\widetilde{\Lambda}(l+1)} d t e^{-t^{2}} \\
& +\sqrt{\operatorname{var}(H)} O\left[\left(R \tilde{b}+R^{2} \widetilde{\Lambda}\right) \varepsilon_{N}\right] \\
& \geq \sqrt{\frac{2 \operatorname{var}(H)}{\pi}} \int_{\tilde{b}}^{\tilde{b}+\tilde{\Lambda}(R+1)} d t(t-\tilde{\Lambda}) e^{-t^{2}} \\
& +\sqrt{\operatorname{var}(H)} O\left[\left(R \tilde{b}+R^{2} \widetilde{\Lambda}\right) \varepsilon_{N}\right] .
\end{aligned}
$$

Since $\Lambda$ and $R$ have so far been free, we choose them such that

$$
\tilde{\Lambda} \rightarrow 0 \quad \text { and } \quad R \tilde{\Lambda} \rightarrow \infty \quad \text { as } \quad N \rightarrow \infty
$$

With this choice, we have

$$
\begin{gathered}
\int_{\tilde{b}}^{\tilde{b}+\widetilde{\Lambda}(R+1)} d t(t-\tilde{\Lambda}) e^{-t^{2}}=\int_{\tilde{b}}^{\infty} d t(t-\tilde{\Lambda}) e^{-t^{2}} \\
+O\left(e^{-R^{2} \widetilde{\Lambda}^{2}}\right)=\frac{1}{2} e^{-\tilde{b}^{2}}+O(\widetilde{\Lambda})+O\left(e^{-R^{2} \widetilde{\Lambda}^{2}}\right)
\end{gathered}
$$

We specify our choice of $\widetilde{\Lambda}$ and $R$ as

$$
\widetilde{\Lambda}=\ln ^{-1} N \quad \text { and } \quad R=\ln ^{2} N
$$

in full accordance with Eq. (E22) (note that this choice is not unique). With these $\widetilde{\Lambda}$ and $R, e^{-R^{2} \widetilde{\Lambda}^{2}}=e^{-\ln ^{2} N}=o(\tilde{\Lambda})$ as $N \rightarrow \infty$; therefore, we can absorb the second $O$ term into the first $O$ term in Eq. (E23). Then, substituting Eq. (E23) into Eq. (E21), we arrive at

$$
\begin{aligned}
\mathfrak{E} \geq & \frac{1}{2} \sqrt{\frac{2 \operatorname{var}(H)}{\pi}}\left[e^{-\tilde{b}^{2}}+O\left(\ln ^{-1} N\right)+O\left(\varepsilon_{N} \tilde{b} \ln ^{2} N\right)\right. \\
& \left.+O\left(\varepsilon_{N} \ln ^{3} N\right)\right]
\end{aligned}
$$

$$
\begin{aligned}
= & \frac{1}{2} \sqrt{\frac{2 \operatorname{var}(H)}{\pi}}\left[e^{-\tilde{b}^{2}}+O\left(\ln ^{-1} N\right)+O\left(N^{-1 / 2} \ln ^{2 \mathfrak{d}+3} N\right)\right. \\
& \left.+O\left(\tilde{b} N^{-1 / 2} \ln ^{2 \mathfrak{d}+2} N\right)\right] .
\end{aligned}
$$

Since, for sufficiently large $N, N^{-1 / 2} \ln ^{2 \mathfrak{d}+3} N=o\left(\ln ^{-1} N\right)$, we can absorb the second $O$ term into the first $O$ term in Eq. (E26), thereby obtaining

$$
\begin{aligned}
\mathfrak{E} \geq & \frac{1}{2} \sqrt{\frac{2 \operatorname{var}(H)}{\pi}}\left[e^{-\tilde{b}^{2}}+O\left(\ln ^{-1} N\right)\right. \\
& \left.+O\left(\tilde{b} N^{-1 / 2} \ln ^{2 \mathfrak{d}+2} N\right)\right] .
\end{aligned}
$$

When $|\tilde{b}| \leq \ln ^{4 / 5} N$, by the same logic as above, the second $O$ term in Eq. (E27) can be absorbed into the first $O$ term, producing

$$
\mathfrak{E} \geq \frac{1}{2} \sqrt{\frac{2 \operatorname{var}(H)}{\pi}}\left[e^{-\tilde{b}^{2}}+O\left(\ln ^{-1} N\right)\right]
$$

To estimate $\mathfrak{E}$ in the $|\tilde{b}| \leq \ln ^{4 / 5} N$ range more precisely, let us find an upper-bound for $\mathfrak{E}$ akin to the bound (E28). To 
that end, we divide the decomposition in Eq. (E17) as

$$
\begin{aligned}
\mathfrak{E}= & \sum_{l=0}^{R} \sum_{\Lambda l \leq E_{i}-b<\Lambda(l+1)} q_{i}\left(E_{i}-\langle H\rangle\right) \\
& +\sum_{l=R+1}^{\infty} \sum_{\Lambda \leq E_{i}-b<\Lambda(l+1)} q_{i}\left(E_{i}-\langle H\rangle\right),
\end{aligned}
$$

with $R$ and $\Lambda$ satisfying the conditions in Eq. (E22).

We first deal with the second term in Eq. (E29). To do so, recall that our system has exponentially decaying correlations, and therefore Theorem 4.2 in Ref. [79] applies. It states that for arbitrary $\mathfrak{d}$-dimensional lattices with exponentially decaying correlations, there exists a constant $\aleph>$
0 such that whenever $|E-\langle H\rangle|>\aleph \sqrt{\operatorname{var}(H)}$ the function

$$
\bar{J}(E)= \begin{cases}\int_{E}^{\infty} d E^{\prime} q\left(E^{\prime}\right) & \text { when } E>\langle H\rangle+\aleph \sqrt{\operatorname{var}(H)}, \\ \int_{-\infty}^{E} d E^{\prime} q\left(E^{\prime}\right) & \text { when } E<\langle H\rangle-\aleph \sqrt{\operatorname{var}(H)}\end{cases}
$$

satisfies

$$
\bar{J}(E) \leq \aleph_{0} \exp \left[-\widetilde{\aleph}\left(\frac{(E-\langle H\rangle)^{2}}{2 \operatorname{var}(H)}\right)^{1 /(\mathfrak{d}+1)}\right],
$$

where $\widetilde{\kappa}>0$ and $\aleph_{0}>0$ are some constants. The bounds provided in Ref. [79] are a bit tighter, but the bound (E31) will be sufficient for our needs here.

Now, turning to the second sum in Eq. (E29), we write

$$
\begin{aligned}
\sum_{l=R+1}^{\infty} \sum_{\Lambda \leq E_{i}-b<\Lambda(l+1)} q_{i}\left(E_{i}-\langle H\rangle\right)= & \int_{b+(R+1) \Lambda}^{\infty} d E(E-\langle H\rangle) q(E) \leq \int_{b+R \Lambda}^{\infty} d E(E-\langle H\rangle) q(E)=\left.(E-\langle H\rangle) \bar{J}(E)\right|_{\infty} ^{b+R \Lambda} \\
& +\int_{b+R \Lambda}^{\infty} d E \bar{J}(E)=(b-\langle H\rangle+R \Lambda) \bar{J}(b+R \Lambda)+\int_{b+R \Lambda}^{\infty} d E \bar{J}(E),
\end{aligned}
$$

where in the second line we noted that $q(E) d E=-d \bar{J}(E)$ and performed integration by parts.

Now, keeping mind that $-\ln ^{4 / 5} N \leq \tilde{b} \leq \ln ^{4 / 5} N$ and, according to Eq. (E22), $R \widetilde{\Lambda}=\ln N$, we have that $\tilde{b}+R \tilde{\Lambda}>0$ and $\tilde{b}+R \tilde{\Lambda} \approx \ln N$, which means that $b+R \Lambda-\langle H\rangle \approx \ln N \sqrt{2 \operatorname{var}(H)}$. Hence, inequality (E31) applies, and therefore we can write

$$
\begin{aligned}
\frac{\sum_{l=R+1}^{\infty} \sum_{\Lambda l \leq E_{i}-b<\Lambda(l+1)} q_{i}\left(E_{i}-\langle H\rangle\right)}{\aleph_{0} \sqrt{2 \operatorname{var}(H)}} & \leq(\tilde{b}+R \widetilde{\Lambda}) \exp \left[-\widetilde{\aleph}(\tilde{b}+R \widetilde{\Lambda})^{2 /(\mathfrak{d}+1)}\right]+\int_{\tilde{b}+R \widetilde{\Lambda}}^{\infty} d x e^{-\widetilde{\aleph} x^{2 /(\mathfrak{d}+1)}} \\
& =(\tilde{b}+R \widetilde{\Lambda}) \exp \left[-\widetilde{\aleph}(\tilde{b}+R \widetilde{\Lambda})^{2 /(\mathfrak{d}+1)}\right]+\frac{\mathfrak{d}+1}{2 \widetilde{\aleph}^{(\mathfrak{d}+1) / 2}} \Gamma\left(\frac{\mathfrak{d}+1}{2}, \widetilde{\aleph}(\tilde{b}+R \widetilde{\Lambda})^{2 /(\mathfrak{d}+1)}\right),
\end{aligned}
$$

where $\Gamma(x, y)$ is the incomplete $\Gamma$ function.

For $y \gg 1, \Gamma(x, y)=y^{x-1} e^{-y}\left[1+O\left(y^{-1}\right)\right]$. Therefore, the second term in the last line of Eq. (E33) is

$$
O\left\{(\tilde{b}+R \tilde{\Lambda})^{(\mathfrak{d}-1) /(\mathfrak{d}+1)} \exp \left[-\tilde{\aleph}(\tilde{b}+R \tilde{\Lambda})^{2 /(\mathfrak{d}+1)}\right]\right\}
$$

which means that

$$
\begin{aligned}
& \frac{\sum_{l=R+1}^{\infty} \sum_{\Lambda l \leq E_{i}-b<\Lambda(l+1)} q_{i}\left(E_{i}-\langle H\rangle\right)}{\sqrt{2 \operatorname{var}(H)}} \\
& \quad=O\left\{(\tilde{b}+R \tilde{\Lambda}) \exp \left[-\tilde{\aleph}(\tilde{b}+R \tilde{\Lambda})^{2 /(\mathfrak{d}+1)}\right]\right\} .
\end{aligned}
$$

For $N \rightarrow \infty$, since $\tilde{b}+R \tilde{\Lambda} \approx \ln N \rightarrow \infty$, the right-hand side of Eq. (E34) decays to zero. However, when $\mathfrak{d}=3$, it starts becoming small only when $N \gtrsim 10^{6}$ (which is for $\widetilde{\aleph}=1$, and for smaller $\widetilde{\aleph}$ it is even slower); for $\mathfrak{d}=1,2$, it becomes small much earlier. To obtain a "safe" estimate for the $O$ term in Eq. (E34), we note that

$$
\begin{aligned}
(\tilde{b} & +R \widetilde{\Lambda})^{2} \exp \left[-\widetilde{\aleph}(\tilde{b}+R \widetilde{\Lambda})^{2 /(\mathfrak{d}+1)}\right]=\frac{1}{\widetilde{\aleph}^{1 /(\mathfrak{d}+1)}} \frac{x^{\mathfrak{d}+1}}{e^{x}} \\
& \leq \frac{1}{\widetilde{\aleph}^{(1 /(\mathfrak{d}+1)}}\left(\frac{\mathfrak{d}+1}{e}\right)^{\mathfrak{d}+1}=O(1),
\end{aligned}
$$

where we have introduced $x=\widetilde{\aleph}(\tilde{b}+R \widetilde{\Lambda})^{2 /(\mathfrak{d}+1)}$ for transparency. Equation (E35) means that the $O$ term in Eq. 
(E34) is $O\left[(\tilde{b}+R \tilde{\Lambda})^{-1}\right]=O\left(\ln ^{-1} N\right)$; thus,

$$
\sum_{l=R+1}^{\infty} \sum_{\Lambda l \leq E_{i}-b<\Lambda(l+1)} q_{i}\left(E_{i}-\langle H\rangle\right) \leq \sqrt{\operatorname{var}(H)} O\left(\ln ^{-1} N\right) .
$$

Substituting Eq. (E36) into Eq. (E29), we obtain

$$
\begin{aligned}
\mathfrak{E}= & \sum_{l=0}^{R} \sum_{\Lambda l \leq E_{i}-b<\Lambda(l+1)} q_{i}\left(E_{i}-\langle H\rangle\right)+\sqrt{\operatorname{var}(H)} O\left(\ln ^{-1} N\right) \\
\leq & \sum_{l=0}^{R}[b-\langle H\rangle+\Lambda(l+1)] \sum_{\Lambda l \leq E_{i}-b<\Lambda(l+1)} q_{i} \\
& +\sqrt{\operatorname{var}(H)} O\left(\ln ^{-1} N\right),
\end{aligned}
$$

which, keeping in mind Eqs. (E14), (E18), and (E20), we transform into

$$
\begin{aligned}
\frac{\mathfrak{E}}{\sqrt{2 \operatorname{var}(H)} \leq} & \frac{1}{\sqrt{\pi}} \sum_{l=0}^{R}[\tilde{b}+\tilde{\Lambda}(l+1)] \int_{\tilde{b}+\tilde{\Lambda} l}^{\tilde{b}+\tilde{\Lambda}(l+1)} d t e^{-t^{2}} \\
& +O\left(R^{2} \widetilde{\Lambda} \varepsilon_{N}\right)+O\left(R \tilde{b} \varepsilon_{N}\right)+O\left(\ln ^{-1} N\right) \\
\leq & \frac{1}{\sqrt{\pi}} \sum_{l=0}^{R} \int_{\tilde{b}+\tilde{\Lambda} l}^{\tilde{b}+\tilde{\Lambda}(l+1)} d t(t+\tilde{\Lambda}) e^{-t^{2}}+O\left(\ln ^{-1} N\right) \\
& =\frac{1}{\sqrt{\pi}} \int_{\tilde{b}}^{\tilde{b}+\tilde{\Lambda}(R+1)} d t(t+\tilde{\Lambda}) e^{-t^{2}}+O\left(\ln ^{-1} N\right) .
\end{aligned}
$$

Proceeding as in Eq. (E23) and taking into account Eq. (E24), we can rewrite Eq. (E37) as

$$
\mathfrak{E} \leq \frac{1}{2} \sqrt{\frac{2 \operatorname{var}(H)}{\pi}}\left[e^{-\tilde{b}^{2}}+O\left(\ln ^{-1} N\right)\right] .
$$

Thus, when $|\tilde{b}| \leq \ln ^{4 / 5} N$, Eqs. (E28) and (E38) show that

$$
\mathfrak{E}=\frac{1}{2} \sqrt{\frac{2 \operatorname{var}(H)}{\pi}}\left[e^{-\tilde{b}^{2}}+O\left(\ln ^{-1} N\right)\right] .
$$

Substitution of Eq. (E39) into Eq. (E16) produces

$$
\frac{\mathcal{C}}{\mathcal{F}}=\frac{2}{\pi} \frac{\left[e^{-\tilde{b}^{2}}+O\left(\ln ^{-1} N\right)\right]^{2}}{1-(\operatorname{erf} \tilde{b})^{2}}\left[1+O\left(\varepsilon_{N} \operatorname{erf} \tilde{b}\right)+O\left(\varepsilon_{N}^{2}\right)\right]
$$

where we used the fact that $\mathcal{F}=\beta^{4} \operatorname{var}(H)$.

Equation (E40) resembles Eq. (36), and shows that the maximum of $\mathcal{C} / \mathcal{F}$ over the boundary position, as quantified by $\tilde{b}$, is reached for some small $\tilde{b}$. To be more specific, Taylor-expanding Eq. (E40) around $\tilde{b}=0$, and considering only $\tilde{b} \ll 1$, we see that

$$
\frac{\mathcal{C}}{\mathcal{F}}=\frac{2}{\pi}\left[1-2 \tilde{b}^{2}(1-2 / \pi)+O\left(\ln ^{-1} N\right)+O\left(\tilde{b}^{3}\right)\right] .
$$

This implies (i) that some $\tilde{b}^{2}=O\left(\ln ^{-1 / 2} N\right)$ yields the maximum

$$
\max _{\tilde{b}} \frac{\mathcal{C}}{\mathcal{F}}=\frac{2}{\pi}+O\left(\ln ^{-1} N\right)
$$

and (ii) that $\mathcal{C} / \mathcal{F}$ is robust with regard to variations of the bin boundary around its optimal position, in the sense discussed in Secs. IV A and IV B.

\section{APPENDIX F: ENERGY DISTRIBUTION OF CRITICAL LATTICE SYSTEMS}

In this section, we show that at a finite-temperature phase transition point, the energy distribution of a translation-invariant, finite-range quantum lattice is Gaussian when the critical exponent $\alpha=0$ ( $\alpha$ is the exponent corresponding to the specific heat; see Sec. IV and Appendix F 2), albeit with a larger variance as compared with the noncritical case (see Appendices E and F 1). When $\alpha>0$, we show that the distribution is unimodal with exponentially decaying tails but not in general Gaussian (Appendix F 2 b). The section starts with a derivation of the general formalism for obtaining the energy distributions, then, in Appendix F 1, we apply the formalism to the known case of noncritical systems, deriving results consistent with the many-body Berry-Esseen theorem $[59,60]$ presented in Appendix E. Finally, in Appendix F2, we analyze the energy distribution for critical systems.

To understand what the energy distribution near the critical point looks like, we will invoke Lemma 12 from Ref. [70] (see also Theorem III.4.15 in Ref. [61]) stating that the cumulative density of states of a translationally invariant, finite-range lattice in arbitrary spatial dimensions is an exponential of the canonical entropy of the lattice. To cast this in more precise terms, we first have to introduce some notation. Fixing periodic boundary conditions, let $H_{N}$ denote the translation-invariant Hamiltonian of an $N$-site lattice $(N \gg 1)$. Then, for an arbitrary translationinvariant state $\Upsilon_{N}$ on the lattice, we define the energy and entropy densities as

$$
\begin{aligned}
& u_{N}\left(\Upsilon_{N}\right)=\frac{1}{N} \operatorname{Tr}\left(H_{N} \Upsilon_{N}\right) \\
& s_{N}\left(\Upsilon_{N}\right)=-\frac{1}{N} \operatorname{Tr}\left(\Upsilon_{N} \ln \Upsilon_{N}\right)
\end{aligned}
$$


Introducing the free energy density,

$$
f_{N}(\beta)=-\frac{T}{N} \ln Z_{N}
$$

where

$$
Z_{N}=\operatorname{Tr} e^{-\beta H_{N}},
$$

we can formulate the variational principle for finite $N$ :

$$
\begin{array}{r}
\min _{\Upsilon_{N}}\left[u_{N}\left(\Upsilon_{N}\right)-T s_{N}\left(\Upsilon_{N}\right)\right]=f_{N}(\beta), \\
\arg \min _{\Upsilon_{N}}\left[u_{N}\left(\Upsilon_{N}\right)-T s_{N}\left(\Upsilon_{N}\right)\right]=\tau_{N}(\beta),
\end{array}
$$

where

$$
\tau_{N}(\beta)=\frac{1}{Z_{N}} e^{-\beta H_{N}}
$$

is the Gibbs state. The finite- $N$ principle is read off straightforwardly from the identity

$$
\left\langle H_{N}\right\rangle_{\Upsilon_{N}}-T S\left(\Upsilon_{N}\right)=F_{N}(\beta)+T S\left[\Upsilon_{N} \| \tau_{N}(\beta)\right]
$$

where $S\left(\Upsilon_{N}\right)=-\operatorname{Tr}\left(\Upsilon_{N} \ln \Upsilon_{N}\right)$ is the von Neumann entropy, $S\left[\Upsilon_{N} \| \tau_{N}(\beta)\right]=\operatorname{Tr}\left\{\Upsilon_{N}\left[\ln \Upsilon_{N}-\ln \tau_{N}(\beta)\right]\right\}$ is the relative entropy, and $F_{N}(\beta)=-T \ln Z_{N}=N f_{N}(\beta)$ is the free energy of the lattice at temperature $T$.

As can be seen, the finite- $N$ situation always yields a thermal state as the unique solution of the minimization in Eq. (F4), and therefore cannot account for secondorder phase transitions at finite temperatures. This is of course in accord with the general understanding that finitetemperature phase transitions appear only in the thermodynamic limit $(N \rightarrow \infty)$. In this limit, the Hilbert space has infinite dimensions, and the simple finite-dimensional argumentation logic breaks down on certain levels. However, as is proven in Refs. [61,62], first of all, the densities

$$
\begin{gathered}
u(\Upsilon)=\lim _{N \rightarrow \infty} u_{N}\left(\Upsilon_{N}\right), \quad s(\Upsilon)=\lim _{N \rightarrow \infty} s_{N}\left(\Upsilon_{N}\right), \quad \text { and } \\
f(\beta)=\lim _{N \rightarrow \infty} f_{N}(\beta)
\end{gathered}
$$

exist and the variational principle still holds:

$$
\inf _{\Upsilon}[u(\Upsilon)-T s(\Upsilon)]=f(\beta),
$$

where the infimum is sought over the set of translationally invariant states. The infimum is delivered by state(s), which we will call "equilibrium state(s)," satisfying the Kubo-Martin-Schwinger (KMS) condition (see, e.g., Refs. $[61,62]$ for a definition; we do not go into the details of it since we do not use that definition in what follows) at inverse temperature $\beta$. The states that satisfy the KMS condition for a given $\beta$ generalize the Gibbs state, always coinciding with it when the Hilbert space is finite dimensional. In infinite dimensions, the KMS state is unique and coincides with the Gibbs state only at or below the critical $\beta_{c}$ if $\beta_{c}$ is finite. Above $\beta_{c}$, the KMS state will generally not be unique, with different KMS states representing different phases (see the discussion in Chap. V in Ref. [80]). When $\beta_{c}=+\infty$, the KMS state is a Gibbs state for arbitrary $\beta<+\infty$; at $\beta_{c}$ (i.e., when the system is in the degenerate ground state), the set of KMS states coincides with the ground eigensubspace. Here, we deal only with lattices for which $\beta_{c}$ is finite (i.e., only thermal phase transitions).

Defining the minimal and maximal possible energy densities as

$$
\begin{aligned}
u_{\min } & :=\lim _{N \rightarrow \infty} \frac{\text { minimal eigenvalue of } H_{N}}{N}, \\
u_{\max } & :=\lim _{N \rightarrow \infty} \frac{\operatorname{Tr} H_{N}}{N d^{N}},
\end{aligned}
$$

where $d$ is the local Hilbert-space dimension of a single node of the lattice, we invoke Lemma 9 from Ref. [70] (also proven in Ref. [61]). It states that for any $u \in\left(u_{\min }, u_{\max }\right]$, there exists a unique $\beta=\beta(u)$ for which at least one equilibrium state at temperature $\beta(u)$ yields energy density $u$. Moreover, the entropy density is

$$
s(u)=\beta(u)[u-f(\beta(u))],
$$

and an analog of the maximal entropy principle holds: $s(u)$ is the highest entropy density among translationally invariant states with energy density $u$.

With this, we are ready to state the result of Lemma 12 in Ref. [70] (which is a clarification and generalization of Theorem III.4.15 in Ref. [61]): if

$$
\left.Q_{N}(u):=\# \text { eigenvalues of } H_{N} \leq u N\right\},
$$

where $\# S$ denotes the cardinality of a finite set $S$, then

$$
\lim _{N \rightarrow \infty} \frac{\ln Q_{N}(u)}{N}=s(u) .
$$

Assuming differentiability of $\ln Q_{N}(u) / N$ with respect to the small parameter $1 / N$, we can write Eq. (F12) as $\ln Q_{N}(u) / N=s(u)+O(1 / N)$, or $Q_{N}(u)=e^{N s(u)+O(1)}$. Furthermore, also assuming differentiability of $s_{N}(u)$ with respect to $1 / N$, we can write $s_{N}(u)=s(u)+O(1 / N)$ (this can be rigorously proven for $1 \mathrm{D}$ and $2 \mathrm{D}$ systems even without assuming analyticity [69]), thereby arriving at

$$
Q_{N}(u)=e^{N s_{N}(u)+O(1)} .
$$

Introducing the density of states [cf. Eq. (18)],

$$
\Omega_{N}(E)=\frac{d Q_{N}(u)}{N d u}
$$


where $E=N u$, we obtain from Eq. (F12)

$$
\Omega_{N}(E)=e^{N s_{N}(u)+O(1)} .
$$

Using this relation, we can write the energy distribution in the sense of Eq. (20) as

$$
q_{N}(E)=\frac{1}{Z_{N}} e^{-\beta N\left[u-T s_{N}(u)\right]+O(1)}=e^{\beta N f_{N}(\beta)-\beta N\left[u-T s_{N}(u)\right]+O(1)},
$$

where, again, $E=N u$, and to obtain the second equality, we used Eq. (F2). The normalization condition thus takes the form

$$
\sum_{n} \frac{e^{-\beta E_{n}}}{Z_{N}}=1=\int_{u_{\min }^{(N)}}^{u_{\max }^{(N)}} d(u N) e^{\beta N f_{N}(\beta)-\beta N\left[u-T_{N}(u)\right]+O(1)} .
$$

Regardless of what the exact form of $q_{N}(E)$ is, by construction it satisfies

$$
\int d(u N) q_{N}(u N) u=u_{N}(\beta),
$$

$$
\int d(u N) q_{N}(u N)\left[u-u_{N}(\beta)\right]^{2}=\operatorname{var} u=\beta^{-2} N^{-1} c_{N}(\beta) .
$$

Fixing an arbitrary $\beta_{0} \leq \beta_{c}$ we observe that the infimum in Eq. (F8) is given by the Gibbs state at temperature $T_{0}$ [see the discussion below Eq. (F8)], and since $N$, however large, is finite, the infimum will be a minimum. Therefore, in the vicinity of $u_{N}\left(\tau_{N}\left(\beta_{0}\right)\right)$ [which we simply call $\left.u_{N}\left(\beta_{0}\right)\right]$, we can write

$$
u-T_{0} s_{N}(u)=f_{N}\left(\beta_{0}\right)+\left.\frac{d^{2}\left[u-T_{0} s_{N}(u)\right]}{d u^{2}}\right|_{u=u_{N}\left(\beta_{0}\right)} \frac{\left[u-u_{N}\left(\beta_{0}\right)\right]^{2}}{2}+\left.\frac{d^{3}\left[u-T_{0} s_{N}(u)\right]}{d u^{3}}\right|_{u=u_{N}\left(\beta_{0}\right)} \frac{\left[u-u_{N}\left(\beta_{0}\right)\right]^{3}}{6}+\cdots .
$$

Writing the double derivative in this formula as

$$
\frac{d^{2}\left[u-T_{0} s_{N}(u)\right]}{d u^{2}}=-T_{0} \frac{d^{2} s_{N}(u)}{d u^{2}}=-T_{0} \frac{d}{d u} \frac{d s_{N}(u)}{d u}
$$

and noting that $d s_{N}(u) / d u=\beta(u)$, we find that

$$
\frac{d^{2}\left[u-T_{0} S_{N}(u)\right]}{d u^{2}}=\frac{T_{0}}{T(u)^{2}} \frac{d T(u)}{d u}=\frac{T_{0}}{T(u)^{2}} \frac{1}{c_{N}[\beta(u)]},
$$

where $c_{N}(\beta)$ is the specific heat of the $N$-site lattice at the inverse temperature $\beta$ :

$$
c_{N}(\beta):=\frac{d u_{N}\left(\tau_{N}(\beta)\right)}{d T} .
$$

Observing that

$$
\beta\left(u_{N}\left(\beta_{0}\right)\right)=\beta_{0},
$$

we thus obtain

$$
K_{2}:=\left.\frac{d^{2}\left[u-T_{0} s_{N}(u)\right]}{d u^{2}}\right|_{u=u_{N}\left(\beta_{0}\right)}=\frac{\beta_{0}}{2 c_{N}\left(\beta_{0}\right)} .
$$

The cubic term is obtained by differentiating Eq. (F22):

$$
\frac{d^{3}\left[u-T_{0} s_{N}(u)\right]}{d u^{3}}=\left.\frac{T_{0} \beta(u)^{4}}{c_{N}(\beta(u))^{3}} \frac{d c_{N}(\beta)}{d \beta}\right|_{\beta=\beta(u)}-\frac{2 T_{0} \beta(u)^{3}}{c_{N}(\beta(u))^{2}},
$$

so

$$
\begin{aligned}
K_{3} & :=\left.\frac{d^{3}\left[u-T_{0} s_{N}(u)\right]}{d u^{3}}\right|_{u=u_{N}\left(\beta_{0}\right)} \\
& =\left.\frac{\beta_{0}^{3}}{6 c_{N}\left(\beta_{0}\right)^{3}} \frac{d c_{N}(\beta)}{d \beta}\right|_{\beta=\beta_{0}}-\frac{\beta_{0}^{2}}{3 c_{N}\left(\beta_{0}\right)^{2}} .
\end{aligned}
$$

The higher-order terms in Eq. (F20) $\left(K_{j \geq 4}\right)$ can be obtained by further differentiating Eq. (F26).

Separating the $u$-dependent part of $O(1)$ in Eq. (F16) as

$$
\text { const }+K_{1}\left[u-u_{N}\left(\beta_{0}\right)\right]+\sum_{j \geq 2} \frac{\zeta_{j}}{j !}\left[u-u_{N}\left(\beta_{0}\right)\right]^{j}
$$

where $K_{1}$ and all $\zeta_{j}$ 's are $O(1)$ (since they depend only on intensive quantities), we can write Eq. (F16) as 


$$
q_{N}(E)=K \exp \left(K_{1}\left[u-u_{N}\left(\beta_{0}\right)\right]-\frac{1}{2}\left(N \beta_{0} K_{2}-\zeta_{2}\right)\left[u-u_{N}\left(\beta_{0}\right)\right]^{2}-\sum_{j \geq 3} \frac{1}{j !}\left(N \beta_{0} K_{j}-\zeta_{j}\right)\left[u-u_{N}\left(\beta_{0}\right)\right]^{j}\right),
$$

where, as usual, $E=u N$. $K$ is a $u$-independent quantity that absorbs all $u$-independent quantities; it is nothing but the normalization factor for $q_{N}(E)$.

\section{Away from criticality: $\boldsymbol{\beta}_{\mathbf{0}}<\boldsymbol{\beta}_{\boldsymbol{c}}$}

When the lattice is away from criticality, $c_{N}(\beta)$ and its derivatives are finite (i.e., do not scale with $N$ ). Therefore, var $u \propto \beta^{-2} N^{1}$, meaning that when deviating from the average, $\langle u\rangle$, by much more than one standard deviation [but $o(\sqrt{N})$ ], $u-\langle u\rangle$ remains $o(1)$. Hence, up to rather far into the tails of the distribution, the quadratic term in the exponent in Eq. (F29) dominates the higher-order terms. Ignoring those higher-order terms and combining the linear and quadratic terms, and absorbing the resulting $u$-independent $e^{K_{2}^{2} c_{N}\left(\beta_{0}\right) /\left(2 \beta_{0}^{2} N\right)}$ into $K$, we find

$$
q_{N}(E)=K \exp \left(-\frac{\beta_{0}^{2} N}{2 c_{N}\left(\beta_{0}\right)}\left[u-\widetilde{u}_{N}\left(\beta_{0}\right)\right]^{2}\right),
$$

where

$$
\tilde{u}_{N}\left(\beta_{0}\right)=u_{N}\left(\beta_{0}\right)+\frac{K_{1} c_{N}\left(\beta_{0}\right)}{\beta_{0}^{2} N} .
$$

Noticing that the difference between $\tilde{u}_{N}\left(\beta_{0}\right)$ and $u_{N}\left(\beta_{0}\right)$ is only proportional to $1 / N$, meaning that $\widetilde{\left\langle H_{N}\right\rangle_{\beta_{0}}}-$ $\left\langle H_{N}\right\rangle_{\beta_{0}}=O(1) c_{N}=O\left(c_{N}\right)$ (which is negligible when $N \gg 1$ ), and rescaling $u$ to $E=N u$, we find that

$$
q_{N}(E)=K \exp \left(-\frac{\left[E-\left\langle H_{N}\right\rangle_{\beta_{0}}+O\left(c_{N}\right)\right]^{2}}{2 \operatorname{var}\left(H_{N}\right)_{\beta_{0}}}\right) .
$$

Our result in this subsection thus complements the manybody Berry-Esseen theorem (Theorem 2 in Appendix E). In particular, the fact that the tails of $q_{N}(E)$ decay exponentially [Eq. (F32)] cannot be directly deduced from the many-body Berry-Esseen theorem; in this sense, our result directly connects to Theorem 4.2 in Ref. [79] (discussed in Appendix E) by strengthening it for the particular case of translationally invariant lattices.

Equation (F32) reflects the "common wisdom" that the thermal state is located in a typical subset of energy levels of width $O\left(\sqrt{\operatorname{var}\left(H_{N}\right)_{\beta_{0}}}\right)=O(\sqrt{N})$, centered at the average energy; the energy levels within the typical subset have approximately equal probabilities, so the entropy of the state is essentially the logarithm of the number of energy levels in the subset [which we see by invoking Eq. (F15) and noting that the number of energy levels in the typical subset is proportional to $\left.\sqrt{N} e^{N s_{N}\left(\beta_{0}\right)}\right]$.

\section{At criticality: $\beta_{0}=\beta_{c}$}

The specific heat of a critical lattice diverges with $N$, and even more divergent are its derivatives. This necessitates a careful bookkeeping of all the terms in the series in Eq. (F29). To do so, we need to find how the specific heat and its temperature derivatives scale at criticality.

First, we recall that as the system approaches the critical temperature, with the approach being parameterized by

$$
t=\frac{\beta-\beta_{c}}{\beta_{c}} \ll 1,
$$

the specific heat and correlation length $(\xi)$ in the thermodynamic limit scale as

$$
c_{\infty}(\beta) \propto|t|^{-\alpha},
$$

$$
\xi(\beta) \propto|t|^{-v}
$$

where $1>\alpha \geq 0$ and $v>0$ are the corresponding critical exponents. When $\alpha=0$ (e.g., in the 2D Ising model),

$$
c_{\infty}(\beta) \propto \ln |t|^{-1}
$$

When $N$ is finite, neither $\xi(\beta)$ nor $c_{N}(\beta)$ can diverge as $t \rightarrow 0$. In this case, since the correlation length diverges in the thermodynamic limit, when the lattice is large but finite, it will simply become proportional to the size of the lattice (for spatial dimension less than 4) [67]. Therefore, in $\mathfrak{d}$ spatial dimensions,

$$
\xi\left(\beta_{c}\right) \propto N^{1 / \mathfrak{d}} .
$$

On the other hand, Eq. (F34) suggests that $c_{N} \propto \xi^{\alpha / \nu}$. Therefore,

$$
c_{N}\left(\beta_{c}\right) \propto N^{\alpha /(\nu \mathfrak{d})}
$$

and when $\alpha=0$,

$$
c_{N}\left(\beta_{c}\right) \propto \ln N
$$

for the proof of this in the case of the 2D Ising model, see Ref. [68]. 
By the same logic, for any $\alpha \geq 0$, we have

$$
\left.\frac{d^{j} c_{N}(\beta)}{d \beta^{j}}\right|_{\beta=\beta_{c}} \propto \beta_{c}^{-j} N^{(\alpha+j) /(v \mathfrak{d})},
$$

since in view of Eqs. (F34) and (F36), $d^{j} c_{\infty}(\beta) / d \beta^{j} \propto$ $\beta^{-j}|t|^{-\alpha-j}$.

Looking into the structure of Eq. (F26) and its derivatives with respect to $u$, we see that the term

$$
\left.\frac{\beta_{c}^{2 j-3}}{c_{N}\left(\beta_{c}\right)^{j}} \frac{d^{j-2} c_{N}(\beta)}{d \beta^{j-2}}\right|_{\beta=\beta_{c}}
$$

is dominant in $K_{j}, j \geq 3$. Therefore,

$$
K_{j} \propto \beta_{c}^{j-1} \begin{cases}(\ln N)^{-j} N^{(j-2) /(\nu \mathfrak{d})} & \text { when } \alpha=0 \\ N^{[j(1-\alpha)+\alpha-2] /(\nu \mathfrak{d})} & \text { when } \alpha>0 .\end{cases}
$$

Taking into account the scaling relation (see, e.g., Ref. [66])

$$
v \mathfrak{d}=2-\alpha,
$$

we can simplify Eq. (F42) to

$$
K_{j} \propto \beta_{c}^{j-1} \begin{cases}(\ln N)^{-j} N^{(j-2) / 2} & \text { when } \alpha=0, \\ N^{j[(1-\alpha) /(2-\alpha)]-1} & \text { when } \alpha>0 .\end{cases}
$$

To determine the regime of validity of the Gaussian approximation to $q_{N}$, we now compare the $j \geq 3$ terms with the quadratic term in Eq. (F29):

$\left(N \beta_{c} K_{2}-\zeta_{2}\right)\left[u-u_{N}\left(\beta_{c}\right)\right]^{2} \gg\left(N \beta_{c} K_{j}-\zeta_{j}\right)\left[u-u_{N}\left(\beta_{c}\right)\right]^{j}$.

Since $N K_{2}$ and all $N K_{j}$ 's diverge with $N$, in the above inequality the $\zeta$ 's, being $O(1)$, are not going to play a role, and therefore, we omit them.

We analyze the $\alpha=0$ and $\alpha>0$ cases separately.

\section{a. Energy distribution for $\boldsymbol{\alpha}=\mathbf{0}$}

In this case, if we take Eq. (F44) into account, Eq. (F45) takes the form

$$
\begin{aligned}
& \beta_{c}(\ln N)^{-1}\left[u-u_{N}\left(\beta_{c}\right)\right]^{2} \gg \beta_{c}^{j-1}(\ln N)^{-j} N^{(j-2) / 2} \\
& \left|u-u_{N}\left(\beta_{c}\right)\right|^{j} \quad \forall j \geq 3,
\end{aligned}
$$

which reduces to

$$
\left|u-u_{N}\left(\beta_{c}\right)\right| \ll \beta_{c}^{-1} \frac{(\ln N)^{1+1 /(j-2)}}{\sqrt{N}} \quad \forall j \geq 3 .
$$

The latter simply means that as long as

$$
\left|u-u_{N}\left(\beta_{c}\right)\right| \lesssim T_{c} \frac{\ln N}{\sqrt{N}},
$$

the quadratic term in Eq. (F29) will dominate the higherorder terms. Noting that for $\alpha=0$ the standard deviation of $u, \sqrt{\operatorname{var} u}$, is proportional to $T_{c} \sqrt{\ln N / N}$, we conclude that the energy distribution is Gaussian up until $O(\sqrt{N})$ standard deviations into the tails.

Just like when away from criticality, the linear term in Eq. (F29) shifts the tip of the distribution function proportional to $T_{c} c_{N}$ [see Eq. (F31)], implying an asymmetry of the distribution as a whole. However, this shift, being proportional to $T_{c} \ln N$, is much less than than the standard deviation $\operatorname{var} E \propto T_{c} \sqrt{N \ln N}$, meaning that the energy distribution, in the energy range in which it is Gaussian, is close to Eq. (E5) [and Eq. (33)].

We quantify the proximity of $q_{N}(E)$ to a Gaussian more precisely for the classical 2D Ising model in Appendix F 3.

\section{b. Energy distribution for $1>\boldsymbol{\alpha}>\mathbf{0}$}

For strictly positive $\alpha$ 's, Eq. (F45) becomes

$$
\begin{aligned}
& \beta_{c} N^{-\alpha /(2-\alpha)}\left[u-u_{N}\left(\beta_{c}\right)\right]^{2} \gg \beta_{c}^{j-1} N^{j[(1-\alpha) /(2-\alpha)]-1} \\
& \left|u-u_{N}\left(\beta_{c}\right)\right|^{j}
\end{aligned}
$$

which leads to

$$
\left|u-u_{N}\left(\beta_{c}\right)\right| \ll T_{c} N^{-(1-\alpha) /(2-\alpha)} .
$$

Taking into account the standard deviation of $u$,

$$
\sqrt{\operatorname{var} u}=T_{c} \sqrt{\frac{c_{N}\left(\beta_{c}\right)}{N}} \propto T_{c} N^{-(1-\alpha) /(2-\alpha)},
$$

we see that the situation with Gaussianity here is trickier than for noncritical lattices or those that are critical but with $\alpha=0$. We see that once we depart one standard deviation from the average, we already find ourselves in a situation where both the quadratic term and the higherorder terms are $O(1)$. However, as long as $\left|E-\left\langle H_{N}\right\rangle_{\beta_{c}}\right| \ll$ $T_{c} N^{1 /(2-\alpha)}, q_{N}(E)$ tends to a Gaussian as $N \rightarrow \infty$.

Lastly, since $\alpha<1, c_{N} / N \rightarrow 0$ as $N \rightarrow \infty$, the shift of the peak of the Gaussian caused by the linear term in Eq. (F29), being proportional to $c_{N} / N$ [see Eq. (F31)], is much less than the variance of $u$, which is proportional to $\sqrt{c_{N} / N}$. Therefore, as in the previous cases, we can ignore that effect, while, of course, keeping in mind that it indicates a certain asymmetry of the overall energy distribution, with the asymmetry becoming more significant the higher the value of $\alpha$.

To sum up, for $\left|E-\left\langle H_{N}\right\rangle_{\beta_{c}}\right| \ll T_{c} N^{1 /(2-\alpha)}, q_{N}(E) \propto$ $\exp \left(-\frac{\left(E-\left\langle H_{N}\right\rangle_{\beta_{c}}\right)^{2}}{2 N c_{N}\left(\beta_{c}\right)}\right)$. That $q_{N}(E)$ significantly deviates from a Gaussian when $\alpha>0$ is not surprising in the light of Appendix F 3.

Furthermore, since $s_{N}(u)$ is a strictly concave function of $u$ (see Theorem III.4.13 in Ref. [61]), and therefore so is $s_{N}(u)-\beta_{c} u$, we see from Eq. (F16) that $-(1 / N) \ln q_{N}(E)$ is a strictly monotonically increasing function once one 
departs from its unique minimum near $\left\langle H_{N}\right\rangle_{\beta_{c}}$. The strict convexity of $s_{N}(u)-\beta_{c} u$ in particular means that once $\left|u-u_{N}\left(\beta_{c}\right)\right|=\Theta(1)$, where $\Theta$ is according to standard asymptotic notation, $q_{N}(N u)=e^{-N \Theta(1)}$. In other words, the distribution $q_{N}(E)$ is unimodal, with exponentially decaying tails.

\section{Classical 2D Ising model at the phase transition}

We now consider the most salient example of a finitetemperature phase transition with $\alpha=0$ - the classical square-lattice 2D Ising model. To make the inevitably complicated analysis as easy as possible, we choose the model to have symmetric, nearest-neighbor couplings, all equal to $1(J=1)$, be at zero magnetic field, and have periodic boundary conditions on both boundaries. In such a case, the free energy per particle in the thermodynamic limit, $f=\lim _{N \rightarrow \infty}(1 / N) F_{N}=-\left(\beta^{-1} / N\right) \ln Z_{N}$ [cf. Eq. (F7)], is given by [75]

$$
\begin{aligned}
f= & -\frac{\ln 2}{2}-\ln \cosh (2 \beta) \\
& +\frac{1}{2 \pi} \int_{0}^{\pi} d \theta \ln \left(1+\sqrt{1-\iota^{2} \cos ^{2} \theta}\right),
\end{aligned}
$$

where

$$
\iota=\frac{2 \sinh (2 \beta)}{\cosh ^{2}(2 \beta)},
$$

with the critical temperature being

$$
\beta_{c}=\frac{\ln (1+\sqrt{2})}{2}
$$

Differentiating $\beta f(\beta)$, we obtain

$$
\begin{aligned}
\frac{\partial(\beta f)}{\partial \beta} & =\lim _{N \rightarrow \infty} \frac{1}{N}\langle H\rangle_{\beta}, \\
\frac{\partial^{2}(\beta f)}{\partial \beta^{2}} & =-\lim _{N \rightarrow \infty} \frac{1}{N}\left\langle\left(H-H_{\beta}\right)^{2}\right\rangle_{\beta} \\
& :=-\lim _{N \rightarrow \infty} \frac{1}{N} E_{2}=-\beta^{-2} c(\beta),
\end{aligned}
$$

where, as before, $c(\beta)$ is the specific heat and

$$
E_{n}:=\left\langle\left(H-H_{\beta}\right)^{n}\right\rangle_{\beta}
$$

is the $n$th central moment. Using the easy-to-derive formula

$$
E_{n+1}=n E_{n-1} E_{2}-\frac{\partial E_{n}}{\partial \beta}, \quad n \geq 2,
$$

we immediately obtain

$$
\frac{\partial^{3}(\beta f)}{\partial \beta^{3}}=\lim _{N \rightarrow \infty} \frac{1}{N}\left\langle\left(H-H_{\beta}\right)^{3}\right\rangle_{\beta}=\lim _{N \rightarrow \infty} \frac{1}{N} E_{3} .
$$

Near the critical temperature [66]

$$
c(\beta) \propto \ln |b|^{-1}
$$

which at criticality translates into [68]

$$
E_{2} \propto N \ln N
$$

Using $E_{3}=-\partial E_{2} / \partial \beta=2 N \beta^{-3} c(\beta)-N \beta^{-2}[\partial c(\beta) / \partial \beta]$, we see that

$$
E_{3} \propto N|b|^{-1}
$$

see Fig. 8(a). This vaguely suggests that at $\beta_{c}, E_{3}$ is nullified for finite but large $N$. However, Eq. (F61) [and therefore Fig. 8(a)] is inconclusive since it is not defined at $\beta_{c}$.

Therefore, to understand what really happens, we need to consider the exact, finite- $N$ solution of the 2D Ising model. This can be easily done with use of the transfer matrices [75], and we use the formulas presented in Refs. $[68,72]$. Feeding these formulas into MATHEMATICA, we find the behavior shown in Fig. 8(a). Denoting the maximal values of $\left|E_{3}\right|$ on both sides of $\beta_{c}$ as

$$
E_{3}^{-}:=\max _{\beta<\beta_{c}} E_{3} \quad \text { and } \quad E_{3}^{+}:=\max _{\beta>\beta_{c}}\left|E_{3}\right|
$$

we find that

$$
E_{3}^{ \pm} \propto N^{3 / 2}
$$

and the distances of the peaks from $\beta_{c}$,

$$
\eta_{-}:=\beta_{c}-\arg \max _{\beta<\beta_{c}} E_{3} \quad \text { and } \quad \eta_{+}:=\arg \max _{\beta>\beta_{c}}\left|E_{3}\right|-\beta_{c} \text {, }
$$

both scale as $N^{-1 / 2}$ :

$$
\eta_{ \pm} \propto N^{-1 / 2}
$$

Importantly, there is a certain assymmetry between the peaks in that they have slightly differing magnitudes and distances from $\beta_{c}$ :

$$
\begin{aligned}
& 0<E_{3}^{+}-E_{3}^{-} \propto N \ln N, \\
& 0<\eta_{-}-\eta_{+} \propto N^{-1} .
\end{aligned}
$$

This suggests that $E_{3}$ is not necessarily zero at $\beta_{c}$, which is of course not surprising as $E_{3}$ is not zero even far away from criticality [see Fig. 8(a)]. At most, $E_{3}\left(\beta_{c}\right)$ may scale 

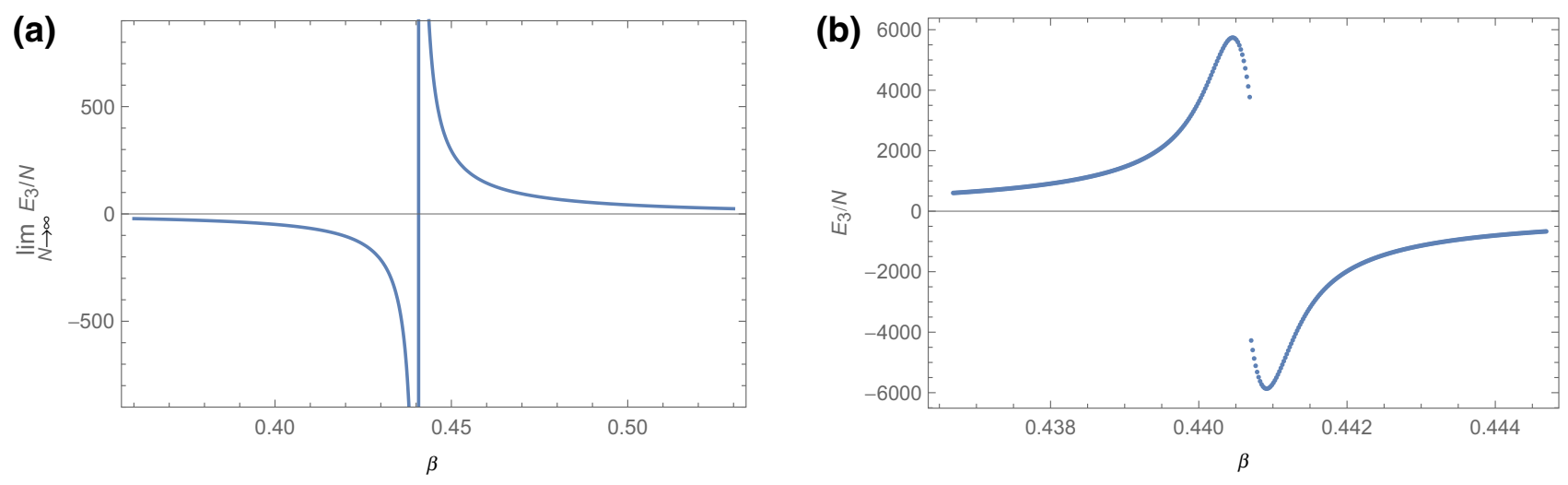

FIG. 8. (a) Density of the third moment of energy of the 2D Ising model in the thermodynamic limit, defined by Eq. (F58), plotted against the inverse temperature $\beta$. (b) The same quantity for $N=10^{6}$, calculated with the exact solution of the $2 \mathrm{D}$ Ising model, plotted against $\beta$.

proportionally with the peaks (i.e., as $N^{3 / 2}$ ); therefore, we can write

$$
E_{3}\left(\beta_{c}\right)=O\left(N^{3 / 2}\right)
$$

Keeping in mind that away from criticality, $E_{3} \propto N$ for the asymmetry, which we quantify by

$$
\mathcal{A}=\frac{\sqrt[3]{E_{3}}}{\sqrt{E_{2}}}
$$

we find that

$$
\begin{aligned}
\mathcal{A}\left(\beta \neq \beta_{c}\right) & \propto N^{-1 / 6}, \\
\mathcal{A}\left(\beta_{c}\right) & \propto \ln ^{-1 / 2} N .
\end{aligned}
$$

In both cases, the asymmetry tends to zero in the thermodynamic limit.

Note that $E_{3}$ peaking near the critical point but not at it fits very well into the general picture drawn above. Indeed, as the temperature approaches $\beta_{c}$, the typical subset of energy levels approaches the less dense region characterized by the increased specific heat [see Eq. (F29), keeping in mind that $K_{2}$ is given by Eq. (F25)]. At some point, part of the subset will be in the critical, "sparse," zone, whereas the other part will be in the noncritical, "dense," zone, which will necessarily make the distribution asymmetric. Then, as one gets even closer to the critical point, most of the typical subset will be contained in the "sparse" zone, thereby mitigating the asymmetry.

To assess the Gaussianity of the energy distribution even further, we invoke the fact that a distribution is Gaussian if and only if its first and second cumulants $\left(\kappa_{1}\right.$ and $\left.\kappa_{2}\right)$ are nonzero whereas all the cumulants starting from the third $\left(\kappa_{k \geq 3}\right)$ are zero. We know that $\kappa_{1}=\langle E\rangle_{\beta}, \kappa_{2}=E_{2}$, and $\kappa_{3}=E_{3}$. Therefore, if we quantify the Gaussianity by the relative weight of a cumulant as compared with $\kappa_{2}$ (i.e., by $\kappa_{k}^{1 / k} / \kappa_{2}^{1 / 2}$ ), then Eq. (F69) for the asymmetry $\mathcal{A}$ already gives an answer for the third cumulant. For the fourth cumulant,

$$
\kappa_{4}=E_{4}-3 E_{2}^{2}=-\frac{\partial E_{3}}{\partial \beta},
$$

where the second equality is due to Eq. (F57), we find numerically by calculating $\partial E_{3} / \partial \beta$ that

$$
\kappa_{4}\left(\beta_{c}\right) \propto N^{2},
$$

which means that

$$
\begin{aligned}
& \left.\frac{\left|\kappa_{4}\right|^{1 / 4}}{\kappa_{2}^{1 / 2}}\right|_{\beta \neq \beta_{c}} \propto N^{-1 / 4}, \\
& \left.\frac{\left|\kappa_{4}\right|^{1 / 4}}{\kappa_{2}^{1 / 2}}\right|_{\beta=\beta_{c}} \propto \ln ^{-1 / 2} N,
\end{aligned}
$$

meaning that the fourth cumulant also becomes asymptotically insignificant in the thermodynamic limit. This shows that, as proved generally in Appendix F 2 a, at criticality the energy distribution of the square-lattice 2D Ising model does indeed tend to a Gaussian.

Lastly, we note from Eqs. (F69) and (F72) that the convergence to Gaussianity at criticality is logarithmic (i.e., much slower than the polynomial convergence away from criticality).

[1] F. Giazotto, T. T. Heikkilä, A. Luukanen, A. M. Savin, and J. P. Pekola, Opportunities for mesoscopics in thermometry and refrigeration: Physics and applications, Rev. Mod. Phys. 78, 217 (2006).

[2] Y. Yue and X. Wang, Nanoscale thermal probing, Nano Rev. 3, 11586 (2012).

[3] M. Mehboudi, A. Sanpera, and L. A. Correa, Thermometry in the quantum regime: Recent theoretical progress, J. Phys. A 52, 303001 (2019). 
[4] L. D. Carlos and F. Palacio, eds., Thermometry at the Nanoscale: Techniques and Selected Applications (The Royal Society of Chemistry, Cambridge, 2016).

[5] U. Marzolino and D. Braun, Precision measurements of temperature and chemical potential of quantum gases, Phys. Rev. A 88, 063609 (2013).

[6] C. Sabin, A. White, L. Hackermuller, and I. Fuentes, Impurities as a quantum thermometer for a Bose-Einstein condensate, Sci. Rep. 4, 6436 (2014).

[7] R. Onofrio, Cooling and thermometry of atomic Fermi gases, Phys. Usp. 59, 1129 (2016).

[8] M. Mehboudi, A. Lampo, C. Charalambous, L. A. Correa, M. A. García-March, and M. Lewenstein, Using Polarons for Sub-NK Quantum Nondemolition Thermometry in a Bose-Einstein Condensate, Phys. Rev. Lett. 122, 030403 (2019).

[9] Q. Bouton, J. Nettersheim, D. Adam, F. Schmidt, D. Mayer, T. Lausch, E. Tiemann, and A. Widera, Single-Atom Quantum Probes for Ultracold Gases Boosted by Nonequilibrium Spin Dynamics, Phys. Rev. X 10, 011018 (2020).

[10] S. Gasparinetti, K. L. Viisanen, O.-P. Saira, T. Faivre, M. Arzeo, M. Meschke, and J. P. Pekola, Fast Electron Thermometry for Ultrasensitive Calorimetric Detection, Phys. Rev. Appl. 3, 014007 (2015).

[11] D. Halbertal, J. Cuppens, M. B. Shalom, L. Embon, N. Shadmi, Y. Anahory, H. R. Naren, J. Sarkar, A. Uri, Y. Ronen, Y. Myasoedov, L. S. Levitov, E. Joselevich, A. K. Geim, and E. Zeldov, Nanoscale thermal imaging of dissipation in quantum systems, Nature 539, 407 (2016).

[12] B. Karimi, F. Brange, P. Samuelsson, and J. P. Pekola, Reaching the ultimate energy resolution of a quantum detector, Nat. Commun. 11, 367 (2020).

[13] K. D. B. Higgins, B. W. Lovett, and E. M. Gauger, Quantum thermometry using the ac stark shift within the rabi model, Phys. Rev. B 88, 155409 (2013).

[14] G. Kucsko, P. C. Maurer, N. Y. Yao, M. Kubo, H. J. Noh, P. K. Lo, H. Park, and M. D. Lukin, Nanometre-scale thermometry in a living cell, Nature 500, 54 (2013).

[15] P. Neumann, I. Jakobi, F. Dolde, C. Burk, R. Reuter, G. Waldherr, J. Honert, T. Wolf, A. Brunner, J. H. Shim, D. Suter, H. Sumiya, J. Isoya, and J. Wrachtrup, High-precision nanoscale temperature sensing using single defects in diamond, Nano Lett. 13, 2738 (2013).

[16] F. Haupt, A. Imamoglu, and M. Kroner, Single Quantum dot as an Optical Thermometer for Millikelvin Temperatures, Phys. Rev. Appl. 2, 024001 (2014).

[17] A. De Pasquale and T. M. Stace, in Thermodynamics in the Quantum Regime: Fundamental Aspects and New Directions, edited by F. Binder, L. A. Correa, C. Gogolin, J. Anders, and G. Adesso (Springer International Publishing, Cham, 2018), p. 503.

[18] L. A. Correa, M. Perarnau-Llobet, K. V. Hovhannisyan, S. Hernández-Santana, M. Mehboudi, and A. Sanpera, Enhancement of low-temperature thermometry by strong coupling, Phys. Rev. A 96, 062103 (2017).

[19] K. V. Hovhannisyan and L. A. Correa, Measuring the temperature of cold many-body quantum systems, Phys. Rev. B 98, 045101 (2018).

[20] S. Seah, S. Nimmrichter, D. Grimmer, J. P. Santos, V. Scarani, and G. T. Landi, Collisional Quantum Thermometry, Phys. Rev. Lett. 123, 180602 (2019).
[21] G. Planella, M. Mehboudi, and A. Acin, Bath-induced correlations lead to sub-shot-noise thermometry precision, arXiv:2001.11812 [quant-ph].

[22] M. Brunelli, S. Olivares, and M. G. A. Paris, Qubit thermometry for micromechanical resonators, Phys. Rev. A 84, 032105 (2011).

[23] M. Brunelli, S. Olivares, M. Paternostro, and M. G. A. Paris, Qubit-assisted thermometry of a quantum harmonic oscillator, Phys. Rev. A 86, 012125 (2012).

[24] S. Jevtic, D. Newman, T. Rudolph, and T. M. Stace, Singlequbit thermometry, Phys. Rev. A 91, 012331 (2015).

[25] A. De Pasquale, K. Yuasa, and V. Giovannetti, Estimating temperature via sequential measurements, Phys. Rev. A 96, 012316 (2017).

[26] V. Cavina, L. Mancino, A. De Pasquale, I. Gianani, M. Sbroscia, R. I. Booth, E. Roccia, R. Raimondi, V. Giovannetti, and M. Barbieri, Bridging thermodynamics and metrology in nonequilibrium quantum thermometry, Phys. Rev. A 98, 050101 (2018).

[27] M. T. Mitchison, T. Fogarty, G. Guarnieri, S. Campbell, T. Busch, and J. Goold, In Situ Thermometry of a Cold Fermi gas via Dephasing Impurities, Phys. Rev. Lett. 125, 080402 (2020).

[28] L. Mancino, M. G. Genoni, M. Barbieri, and M. Paternostro, Nonequilibrium readiness and precision of Gaussian quantum thermometers, Phys. Rev. Res. 2, 033498 (2020).

[29] I. Henao and R. Uzdin, Catalytic transformations with finite-size environments: Applications to cooling and thermometry, arXiv:2010.09070 [quant-ph].

[30] P. P. Potts, J. B. Brask, and N. Brunner, Fundamental limits on low-temperature quantum thermometry with finite resolution, Quantum 3, 161 (2019).

[31] M. R. Jørgensen, P. P. Potts, M. G. A. Paris, and J. B. Brask, Tight bound on finite-resolution quantum thermometry at low temperatures, Phys. Rev. Res. 2, 033394 (2020).

[32] L. A. Correa, M. Mehboudi, G. Adesso, and A. Sanpera, Individual Quantum Probes for Optimal Thermometry, Phys. Rev. Lett. 114, 220405 (2015).

[33] M. G. A. Paris, Achieving the Landau bound to precision of quantum thermometry in systems with vanishing gap, J. Phys. A 49, 03LT02 (2015).

[34] S. Campbell, M. G. Genoni, and S. Deffner, Precision thermometry and the quantum speed limit, Quantum Sci. Technol. 3, 025002 (2018).

[35] A. De Pasquale, D. Rossini, R. Fazio, and V. Giovannetti, Local quantum thermal susceptibility, Nat. Commun. 7, 12782 (2016).

[36] G. De Palma, A. De Pasquale, and V. Giovannetti, Universal locality of quantum thermal susceptibility, Phys. Rev. A 95, 052115 (2017).

[37] H. J. D. Miller and J. Anders, Energy-temperature uncertainty relation in quantum thermodynamics, Nat. Commun. 9, 2203 (2018).

[38] F. M. Spiegelhalder, A. Trenkwalder, D. Naik, G. Hendl, F. Schreck, and R. Grimm, Collisional Stability of ${ }^{4} 0 \mathbf{K}$ Immersed in a Strongly Interacting Fermi Gas of ${ }^{6} \mathrm{Li}$, Phys. Rev. Lett. 103, 223203 (2009).

[39] D. McKay and B. DeMarco, Thermometry with spindependent lattices, New J. Phys. 12, 055013 (2010).

[40] R. Olf, F. Fang, G. E. Marti, A. MacRae, and D. M. Stamper-Kurn, Thermometry and cooling of a Bose gas to 
0.02 times the condensation temperature, Nat. Phys. 11, 720 (2015).

[41] M. Hohmann, F. Kindermann, T. Lausch, D. Mayer, F. Schmidt, and A. Widera, Single-atom thermometer for ultracold gases, Phys. Rev. A 93, 043607 (2016).

[42] R. S. Lous, I. Fritsche, M. Jag, B. Huang, and R. Grimm, Thermometry of a deeply degenerate fermi gas with a bose-einstein condensate, Phys. Rev. A 95, 053627 (2017).

[43] L.-S. Guo, B.-M. Xu, J. Zou, and B. Shao, Improved thermometry of low-temperature quantum systems by a ring-structure probe, Phys. Rev. A 92, 052112 (2015).

[44] V. Mukherjee, A. Zwick, A. Ghosh, X. Chen, and G. Kurizki, Enhanced precision bound of low-temperature quantum thermometry via dynamical control, Commun. Phys. 2, 162 (2019).

[45] A. Recati, P. O. Fedichev, W. Zwerger, J. von Delft, and P. Zoller, Atomic Quantum Dots Coupled to a Reservoir of a Superfluid Bose-Einstein Condensate, Phys. Rev. Lett. 94, 040404 (2005).

[46] S. L. Braunstein and C. M. Caves, Statistical Distance and the Geometry of Quantum States, Phys. Rev. Lett. 72, 3439 (1994).

[47] B. B. Mandelbrot, An outline of a purely phenomenological theory of statistical thermodynamics: I. canonical ensembles, IRE Trans. Inf. Theory 2, 190 (1956).

[48] J. Uffink and J. van Lith, Thermodynamic uncertainty relations, Found. Phys. 29, 655 (1999).

[49] A. W. van der Vaart, Asymptotic Statistics, Vol. 3 (Cambridge University Press, Cambridge, England, 2000).

[50] T. Jahnke, S. Lanéry, and G. Mahler, Operational approach to fluctuations of thermodynamic variables in finite quantum systems, Phys. Rev. E 83, 011109 (2011).

[51] R. G. Gallager, Principles of Digital Communication (Cambridge University Press, New York, 2008).

[52] S. Kullback, Information Theory and Statistics (Dover, New York, 1968).

[53] In that $p_{\alpha}$ cannot be written as $\exp [\mathfrak{K}(\alpha) f(T)+\mathfrak{L}(T)+$ $\mathfrak{M}(\alpha)]$, where $f, \mathfrak{K}, \mathfrak{L}$, and $\mathfrak{M}$ are some functions.

[54] Indeed, it follows from the Bayesian Cramér-Rao bound (also known as the van Trees inequality) [81] that for any estimator, in the $n \rightarrow \infty$ limit, $\Delta T \gtrsim 1 /\left[n \int d T p(T) \mathcal{C}(T)\right]$, where $p(T)$ is the prior distribution of $T$.

[55] E. G. Effros, A matrix convexity approach to some celebrated quantum inequalities, Proc. Natl. Acad. Sci. U. S. A. 106, 1006 (2009).

[56] A. K. Chakravarty, J. B. Orlin, and U. G. Rothblum, Consecutive optimizers for a partitioning problem with applications to optimal inventory groupings for joint replenishment, Oper. Res. 33, 820 (1985).

[57] The solutions to this equation are also guaranteed to be an actual minimum of $\mathcal{D}$, never a maximum. First, $\partial^{2} \mathcal{D} / \partial b_{\alpha} \partial b_{\alpha^{\prime}}=0$ for $\alpha^{\prime} \neq \alpha$. Second, at $b_{\alpha}=\left(\epsilon_{\alpha+1}+\right.$ $\left.\epsilon_{\alpha}\right) / 2$, we have $\partial^{2} \mathcal{D} / \partial b_{\alpha}^{2}=2 \beta^{4}\left(\epsilon_{\alpha+1}-\epsilon_{\alpha}\right) q\left(b_{\alpha}\right) \geqslant 0$ since $q\left(b_{\alpha}\right) \geqslant 0$ by construction and $\epsilon_{\alpha+1} \geqslant \epsilon_{\alpha}$ by hypothesis.

[58] J. P. Keating, N. Linden, and H. J. Wells, Spectra and eigenstates of spin chain hamiltonians, Commun. Math. Phys. 338, 81 (2015).
[59] F. G. S. L. Brandão and M. Cramer, Equivalence of statistical mechanical ensembles for non-critical quantum systems, arXiv:1502.03263 [quant-ph].

[60] F. G. S. L. Brandão, M. Cramer, and M. Guţă, in QIP2015 Conference Talk (2015).

[61] B. Simon, The Statistical Mechanics of Lattice Gases, Vol. 1 (Princeton University Press, Princeton, 1993).

[62] H. Araki, On the equivalence of the KMS condition and the variational principle for quantum lattice systems, Commun. Math. Phys. 38, 1 (1974).

[63] M. B. Hastings, Decay of Correlations in Fermi Systems at Nonzero Temperature, Phys. Rev. Lett. 93, 126402 (2004).

[64] M. Kliesch, C. Gogolin, M. J. Kastoryano, A. Riera, and J. Eisert, Locality of Temperature, Phys. Rev. X 4, 031019 (2014).

[65] T. Farrelly, F. G. S. L. Brandão, and M. Cramer, Thermalization and Return to Equilibrium on Finite Quantum Lattice Systems, Phys. Rev. Lett. 118, 140601 (2017).

[66] M. E. Fisher, The theory of equilibrium critical phenomena, Rep. Prog. Phys. 30, 615 (1967).

[67] E. Brézin, An investigation of finite size scaling, J. Phys. France 43, 15 (1982).

[68] N. S. Izmailian and C.-K. Hu, Exact amplitude ratio and finite-size corrections for the $m \times n$ square lattice Ising model, Phys. Rev. E 65, 036103 (2002).

[69] R. B. Griffiths, A proof that the free energy of a spin system is extensive, J. Math. Phys. 5, 1215 (1964).

[70] M. P. Müller, E. Adlam, L. Masanes, and N. Wiebe, Thermalization and canonical typicality in translation-invariant quantum lattice systems, Commun. Math. Phys. 340, 499 (2015).

[71] T. D. Schultz, D. C. Mattis, and E. H. Lieb, Twodimensional Ising model as a soluble problem of many fermions, Rev. Mod. Phys. 36, 856 (1964).

[72] P. D. Beale, Exact Distribution of Energies in the TwoDimensional Ising Model, Phys. Rev. Lett. 76, 78 (1996).

[73] H. A. Kramers and G. H. Wannier, Statistics of the TwoDimensional ferromagnet. Part I, Phys. Rev. 60, 252 (1941).

[74] L. Onsager, Crystal statistics. I. A two-dimensional model with an order-disorder transition, Phys. Rev. 65, 117 (1944).

[75] R. J. Baxter, Exactly Solved Models in Statistical Mechanics (Academic Press, London, 1982).

[76] V. Giovannetti, S. Lloyd, and L. Maccone, Advances in quantum metrology, Nat. Photonics 5, 222 (2011).

[77] N. K. Langford, R. Sagastizabal, M. Kounalakis, C. Dickel, A. Bruno, F. Luthi, D. J. Thoen, A. Endo, and L. DiCarlo, Experimentally simulating the dynamics of quantum light and matter at deep-strong coupling, Nat. Commun. 8, 1715 (2017).

[78] J. Rubio, J. Anders, and L. A. Correa, Global quantum thermometry, arXiv:2011.13018 [quant-ph].

[79] A. Anshu, Concentration bounds for quantum states with finite correlation length on quantum spin lattice systems, New J. Phys. 18, 083011 (2016).

[80] R. Haag, Local Quantum Physics: Fields, Particles, Algebras (Springer, New York, 1996).

[81] R. D. Gill and B. Y. Levit, Applications of the van Trees inequality: A Bayesian cramér-Rao bound, Bernoulli 1, 59 (1995). 